University of Tennessee Health Science Center

UTHSC Digital Commons

\title{
Use of Compliant Interbody Force Sensing Grafts to Compare Load Sharing Properties of Unidirectional and Bidirectional Multilevel Dynamic Translational Anterior Cervical Plates
}

Jason Roscoe Roberson

University of Tennessee Health Science Center

Follow this and additional works at: https://dc.uthsc.edu/dissertations

Part of the Investigative Techniques Commons

\section{Recommended Citation}

Roberson, Jason Roscoe , "Use of Compliant Interbody Force Sensing Grafts to Compare Load Sharing Properties of Unidirectional and Bidirectional Multilevel Dynamic Translational Anterior Cervical Plates" (2008). Theses and Dissertations (ETD). Paper 357. http://dx.doi.org/10.21007/etd.cghs.2008.0261. 


\title{
Use of Compliant Interbody Force Sensing Grafts to Compare Load Sharing Properties of Unidirectional and Bidirectional Multilevel Dynamic Translational Anterior Cervical Plates
}

\begin{abstract}
INTRODUCTION: Pseudoarthrosis is a relatively rare complication following anterior cervical arthrodesis, and is felt to be related to stress shielding. To address this, dynamic anterior cervical plates have emerged to maintain load sharing as an arthodesis matures. Dynamic plates can translate bidirectionally (allow translation in compressive and tensile loads), or unidirectionally (allow translation in compression, but maintain its position under tensile loads). The objective of this study was to compare the graft load mechanics between three plated conditions during settling using compliant interbody load cells: A static (fixed, non-moving) plate, a unidirectional multi-level translational plate design, and a bidirectional twolevel translational plate design.
\end{abstract}

METHODS: Six fresh human cadaveric cervical spines (C2-T1) were procured and mounted in a programmable testing apparatus and tested in flexion-extension, left-right lateral bending, and left-right axial rotation under displacement control to a load limit of $3.0 \mathrm{Nm}$ or 30 degrees of motion. Four different spine conditions were evaluated: the harvested $(\mathrm{H})$ condition and three types of instrumented conditions containing two-level discectomy and graft at C4-C5 and C5-C6: a unidirectional translational plate, a bidirectional translational plate, and a rigid static plate. Compliant force sensing grafts were placed in the discectomized regions prior to plate application that permitted up to $2 \mathrm{~mm}$ of deformation during compressive loading. The ATLANTIS ${ }^{\circledR}$ Translational cervical plating system (Medtronic Spinal and Biologics, Memphis, TN) was used for the plated spine conditions with fixed-angle screws. Measurements included vertebral motion, applied load and moment, and graft loads. Normalized flexibility data, motion data, and graft load data were compared using a one-way Repeated Measures ANOVA $(p<0.05)$ and SNK tests.

RESULTS: A significant reduction in flexion+extension motion occurred at the operated levels of all instrumented spines compared to the harvested spine. The normalized flexion+extension motion for the unidirectional plate was significantly less than the bidirectional plate. During flexion, the unidirectional plate shortened an average of $2 \mathrm{~mm}$ ( $1 \mathrm{~mm}$ per level). Once shortened, a significant increase in the baseline graft preload occurred.

CONCLUSION: In this study, a compliant interbody load cell was developed and used to determine the graft load properties of three different anterior cervical plate designs: static, unidirectional and bidirectional translational. Both translational plates demonstrated better load-sharing properties than the static plate. The results showed that the unidirectional plate had less motion in flexion/extension, while maintaining a more continuous graft loading than the bidirectional plate. Also, the ratchet mechanism of unidirectional translational plate was validated in the study, where the plate was able to translate under compressive load and maintain its position under tension when ratcheted. The ability to limit motion and maintain compression with a unidirectional dynamic plate may have improved rates of fusion; however, this will need to be evaluated further in an in vivo and/or clinical model.

\section{Document Type}

Thesis

Degree Name

Master of Science (MS) 


\section{Program}

Biomedical Engineering and Imaging

Research Advisor

Denis J DiAngelo, Ph.D.

\section{Keywords}

Anterior plate, spinal fixation, compliant force sensing graft, cervical plate, biomechanics, spinal reconstruction, in-vitro testing

\section{Subject Categories}

Analytical, Diagnostic and Therapeutic Techniques and Equipment | Investigative Techniques | Medicine and Health Sciences 
USE OF COMPLIANT INTERBODY FORCE SENSING GRAFTS TO COMPARE LOAD SHARING PROPERTIES OF UNIDIRECTIONAL AND BIDIRECTIONAL MULTILEVEL DYNAMIC TRANSLATIONAL ANTERIOR CERVICAL PLATES

\author{
A Thesis \\ Presented for \\ The Graduate Studies Council \\ The University of Tennessee \\ Health Science Center
}

\begin{abstract}
In Partial Fulfillment
Of the Requirements for the Degree

Master of Science

In the Joint Graduate Program in Biomedical Engineering and Imaging

From The University of Tennessee

and

The University of Memphis
\end{abstract}

By

Jason Roscoe Roberson

December 2008 
Copyright $(\subseteq) 2008$ by Jason Roscoe Roberson All rights reserved 


\section{DEDICATION}

This thesis is dedicated to my parents, John and Kathryn Roberson, who taught me the meanings of hard work, diligence, and love. 


\section{ACKNOWLEDGEMENTS}

My sincerest gratitude is extended to my advisor Dr. Denis DiAngelo for his help, guidance and mentorship, to my committee Dr. Brian Kelly and Dr. Gladius Lewis, my fellow lab members Brian Morrow, Laura Gilmour, Braham Dhillon, Peter Wong, and Saurabh Kumar for their help with the project, the Medical Education Research Institute for their generosity in the use of their facilities, Medtronic Sofamor Danek for providing project funding, and neurosurgeon Dr. Dean Karahalios for his surgical expertise during device implantation. 


\begin{abstract}
Introduction: Pseudoarthrosis is a relatively rare complication following anterior cervical arthrodesis, and is felt to be related to stress shielding. To address this, dynamic anterior cervical plates have emerged to maintain load sharing as an arthodesis matures. Dynamic plates can translate bidirectionally (allow translation in compressive and tensile loads), or unidirectionally (allow translation in compression, but maintain its position under tensile loads). The objective of this study was to compare the graft load mechanics between three plated conditions during settling using compliant interbody force sensing grafts: A static (fixed, non-moving) plate, a unidirectional multi-level translational plate design, and a bidirectional two-level translational plate design.
\end{abstract}

Methods: Six fresh human cadaveric cervical spines (C2-T1) were procured and mounted in a programmable testing apparatus and tested in flexion-extension, left-right lateral bending, and left-right axial rotation under displacement control to a load limit of $3.0 \mathrm{Nm}$ or 30 degrees of motion. Four different spine conditions were evaluated: the harvested $(\mathrm{H})$ condition and three types of instrumented conditions containing two-level discectomy and graft at C4-C5 and C5-C6: a unidirectional translational plate, a bidirectional translational plate, and a rigid static plate. Compliant force sensing grafts were placed in the discectomized regions prior to plate application that permitted up to $2 \mathrm{~mm}$ of deformation during compressive loading. The ATLANTIS $^{\circledR}$ Translational cervical plating system (Medtronic Spinal and Biologics, Memphis, TN) was used for the plated spine conditions with fixed-angle screws. Measurements included vertebral motion, applied load and moment, and graft loads. Normalized flexibility data, motion data, and graft load data were compared using a one-way Repeated Measures ANOVA $(p<0.05)$ and SNK tests.

Results: A significant reduction in flexion+extension motion occurred at the operated levels of all instrumented spines compared to the harvested spine. The normalized flexion+extension motion for the unidirectional plate was significantly less than the bidirectional plate. During flexion, the unidirectional plate shortened an average of $2 \mathrm{~mm}$ ( $1 \mathrm{~mm}$ per level). Once shortened, a significant increase in the baseline graft preload occurred.

Conclusions: In this study, a compliant interbody force sensing graft was developed and used to determine the graft load properties of three different anterior cervical plate designs: static, unidirectional and bidirectional translational. Both translational plates demonstrated better load-sharing properties than the static plate. The results showed that the unidirectional plate had less motion in flexion/extension, while maintaining a more continuous graft loading than the bidirectional plate. Also, the ratchet mechanism of unidirectional translational plate was validated in the study, where the plate was able to translate under compressive load and maintain its position under tension when ratcheted. The ability to limit motion and maintain compression with a unidirectional dynamic plate may have improved rates of fusion; however, this will need to be evaluated further in an in vivo and/or clinical model. 
TABLE OF CONTENTS

CHAPTER 1. INTRODUCTION .

1.1 Relevant Spine Anatomy and Pathophysiology

1.2 Purpose of the Study .

1.3 Significance of the Study

CHAPTER 2. BACKGROUND

2.1 Definitions of Major Concepts

2.2 Dynamic Plate Design.

2.3 Literature Review

CHAPTER 3. MATERIALS AND METHODS

3.1 Device Design.

3.2 Specimen Preparation.

3.3 Spinal Conditions .

3.4 Test Equipment

3.5 Non-Destructive Testing Protocol 12

3.6 Data Management and Statistical Processing. . . . . . . . . 16

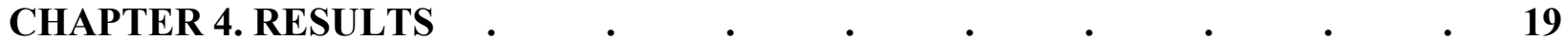

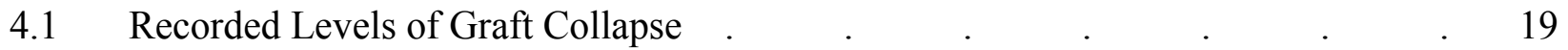

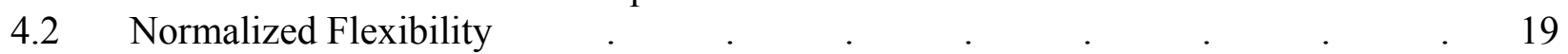

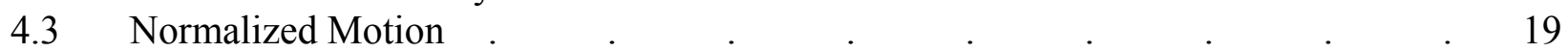

4.4 Motion Segment Unit Percent Contribution . . . . . . . . . 19

4.5 Peak Graft Loads . . . . . . . . . . . . . . 24

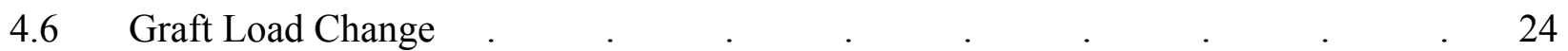

4.7 Graft Preloads . $\quad . \quad$. $\quad . \quad$. $\quad . \quad$. $\quad . \quad . \quad . \quad 27$

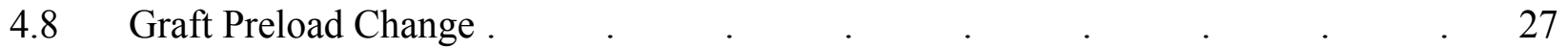

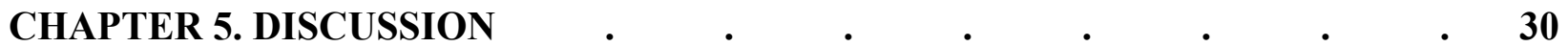

5.1 Inferior versus Superior Level Comparison . . . . . . . . . 31

5.2 Static versus Dynamic Plate Comparison . . . . . . . . 30

5.3 Unidirectional versus Bidirectional Plate Comparison . . . . . 31

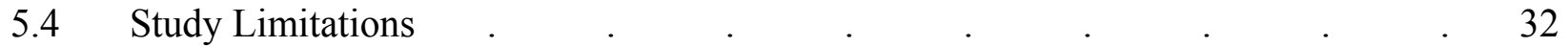

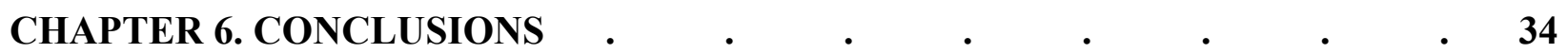

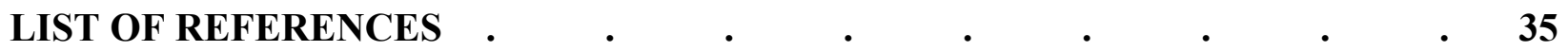

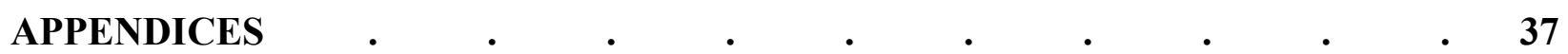

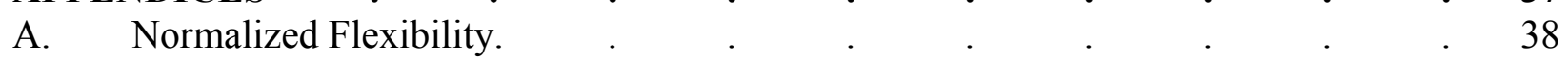

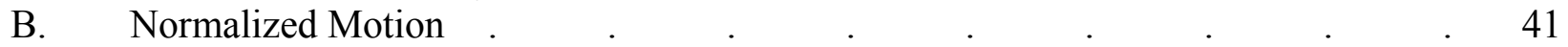

C. Motion Segment Unit Percent Contribution . . . . . . . 45

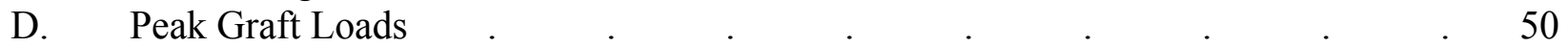




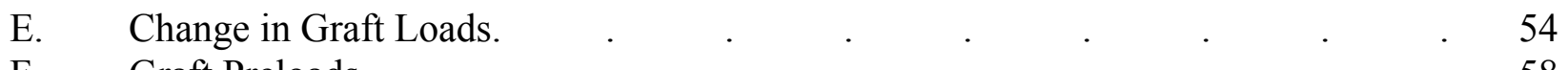

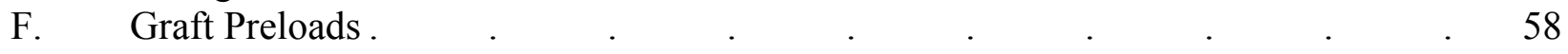

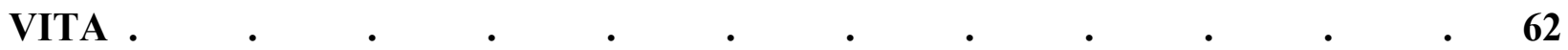




\section{LIST OF TABLES}

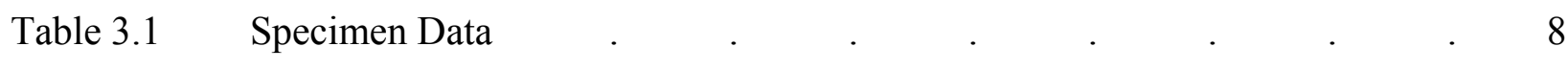

Table 4.1 Observed Unidirectional Plate Collapse $\quad$. $\quad$. $\quad$. . . . 20 


\section{LIST OF FIGURES}

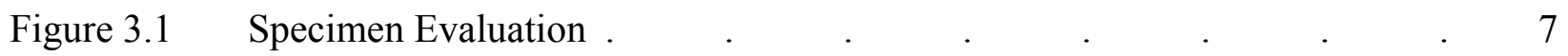

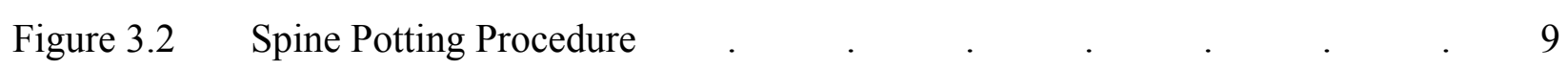

Figure 3.3 Single Actuator Adaptable Programmable Testing Apparatus (SAAPTA) . 11

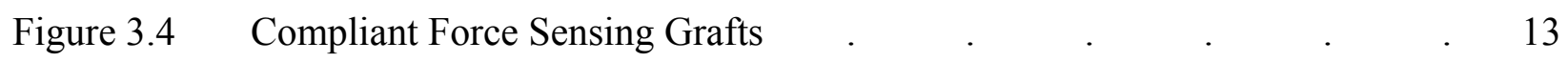

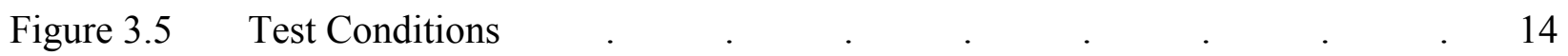

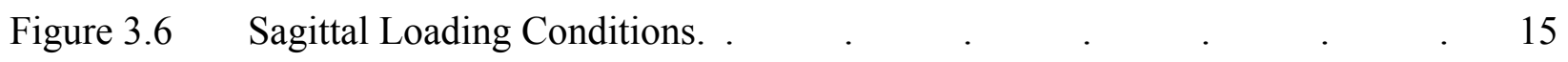

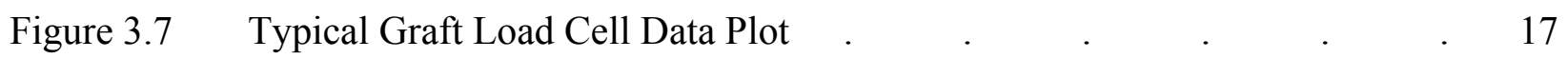

Figure 4.1 Normalized Flexibility for Flexion and Extension $\quad . \quad$. $\quad$. $\quad 21$

Figure 4.2 Normalized Motion for Combined Left and Right Lateral Bending . 22

Figure 4.3 Motion Segment Unit (MSU) Percent Contribution for Combined Flexion

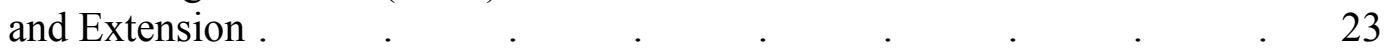

Figure 4.4 Suggested Significance in Peak Graft Loads for Flexion and Extension . 25

Figure 4.5 Combined Superior and Inferior Change in Graft Loads for Flexion

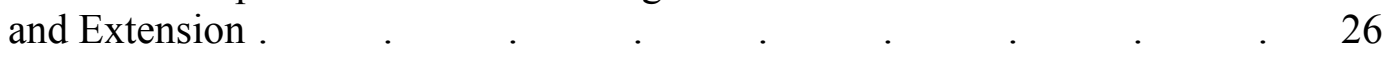

Figure 4.6 Combined Superior and Inferior Graft Preloads for Flexion and Extension . 28

Figure 4.7 Change in Graft Preloads for Flexion and Extension . _ . . . . 29

Figure A.1 Normalized Flexibility for Left and Right Lateral Bending . _ . 39

Figure A.2 Normalized Flexibility for Left and Right Axial Rotation _ . . . $\quad 40$

Figure B.1 Normalized Motion for Flexion and Extension. $\quad$. $\quad$. . . . 42

Figure B.2 Normalized Motion for Left and Right Lateral Bending _ . . 43

Figure B.3 Normalized Motion for Left and Right Axial Rotation _ . . 44

Figure C.1 Motion Segment Unit Percent Contribution for Flexion and Extension $\quad$ • $\quad 46$ 
Figure C.2 Motion Segment Unit Percent Contribution for Left and Right Lateral Bending . . . . . . . . . . .

Figure C.3 Motion Segment Unit Percent Contribution for Left and Right Axial

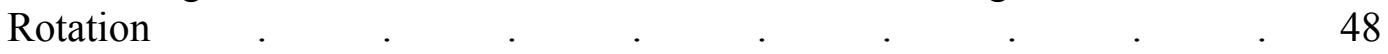

Figure C.4 Motion Segment Unit Percent Contribution for Harvested Condition

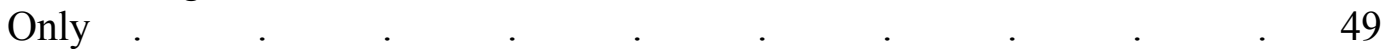

Figure D.1 Peak Graft Loads for Flexion and Extension . _ . . . . . 51

Figure D.2 Peak Graft Loads for Left and Right Lateral Bending _ . 52

Figure D.3 Peak Graft Loads for Left and Right Axial Rotation . _ . 53

Figure E.1 Change in Graft Loads for Flexion and Extension . . . . . 55

Figure E.2 Change in Graft Loads for Left and Right Lateral Bending . 56

Figure E.3 Change in Graft Loads for Left and Right Axial Rotation _ . . 57

Figure F.1 Graft Preloads for Flexion and Extension _ . . . . . . 59

Figure F.2 Graft Preloads for Left and Right Lateral Bending . . . . . . 60

Figure F.3 Graft Preloads for Left and Right Axial Rotation _ . . . . . 61 


\section{CHAPTER 1. INTRODUCTION}

In the 1950s, anterior cervical surgery was first theorized by Dr. Leroy Abbott while under the service of Drs. Bailey and Badgley. Later, Drs. Bailey and Badgley described the approach themselves as well [1]. The methods were then employed in 1958, with the introduction of a horse-shoe shaped bone-graft by Robinson and Smith for enhanced cervical bone fusion [2]. More popularly, it was also used by Cloward, with his introduction of the cylindrical graft for cervical fusion after discectomy [2].

Since then, there have been numerous advances in cervical stabilization techniques. Trauma cases demonstrated a need for further spinal stabilization, leading to the development of the first ventral cervical plate by Bohler in 1964 [3].

There have since been over three decades of research and refinement on the employment and use of anterior cervical plates for spinal stabilization, with the goals being neural structure decompression, immediate stability, reduction of deformity, and creation of successful osseous spinal fusion in a timely manner for cases involving discectomy or corpectomy for degenerative, traumatic, and oncologic disorders. A recent research focus has been to address the incidence of pseudoarthrosis, a complication found following anterior cervical arthrodesis where the bone fusion fails to occur. Stress shielding of the fusion site by the anterior cervical plate is theorized to be the cause, as bone graft settling and subsidence occurs and causes improper load sharing between the plate and the bone graft.

The research documented in this thesis compares recent advances in dynamic anterior cervical plating systems to current standard approved methods, and is intended to help in further understanding of the dynamic plating system.

\subsection{Relevant Spine Anatomy and Pathophysiology}

The human spine is composed of thirty-two to thirty-four bones of which twenty-four are individual vertebrae. Five of them are fused together to form the sacrum in the pelvic region, and three to five are fused to form the coccyx or tailbone. The twenty-four individual vertebrae are

divided into three distinct regions; the upper seven as cervical, the middle twelve as thoracic, and the lower five as lumbar. The seven vertebrae of the cervical spine are further divided into upper $(\mathrm{C} 0-\mathrm{C} 2)$ and lower $(\mathrm{C} 3-\mathrm{C} 7)$ segments. Each vertebrae is separated by the vertebral disc and facet plates along the tops and bottom of the pedicles, forming joint complexes between each vertebrae. The flexible non-calcified disc and the smooth cartilage pads allow translation and rotation at each joint.

The lower cervical segment was the subject of this biomechanical study due to the complexity of the $\mathrm{C} 0-\mathrm{C} 1-\mathrm{C} 2$ joint complex, and the lack of true synovial joints and disc material in the upper segment. In this study, we considered the spinal levels of C2 to T1. Though T1 is designated as a thoracic vertebra, it is transitional in nature, and anatomically similar to the vertebrae of the cervical spine [12]. 
Pseudoarthrosis is a bone fracture disease that may result from fracture or surgical reconstruction that results in a false joint, characterized by pain and motion at the fracture or surgical site [13]. In a surgical sense, pseudoarthrosis refers to the failure of bone to properly fuse post-operatively. During cervical spine fixation surgery, failure of the bone graft to properly fuse causes pseudoarthrosis between the fused levels, and requires intervention. A known cause of post-operative pseudoarthrosis is stress shielding of the fusion site as subsidence occurs during bone remodeling, a trait of static anterior cervical plates. Healing may be achieved by immobilization of the site, or surgery involving internal fixation and/or transplantation of bone to correct the defect [13].

\subsection{Purpose of the Study}

The objectives of this study were as follows: to develop equipment and methodology able to measure graft loads specifically during a graft height reduction, through the use of a compliant load sensing graft system, second, to apply this technology to document the biomechanical properties of three different surgically implanted multi-level anterior cervical plating systems in a cadaver study, including a rigid static plate, a dynamic bidirectional plate, and a unidirectional dynamic plate following a two-level discectomy and simulated spinal fusion procedure; and third, to compare these conditions to the intact control state (harvested), as well as all three plating systems to one another.

\subsection{Significance of the Study}

Cervical plate fixation has been the standard of care for trauma requiring immediate stability for nearly three decades, and has been well studied. Dynamic plates have been incorporated into bone fusion surgery for several decades, and their effects have been well studied. However, dynamic plating systems come with many design features that have not been well studied, or directly compared to one another. Further understanding of the biomechanical properties of dynamic plates involving direct graft force measurements is needed in order to forward dynamic plating research.

The use of dynamic cervical plates has emerged to address stress shielding. These plates allow limited translational and rotational movement between the fused structures. Clinical data have shown that dynamic plates reduce the occurrence of pseudarthrosis, increase spinal fusion rates, as well as reduce the rate of implant complications [5]. It is theorized that dynamic plates do this with increased load sharing capabilities, preventing stress shielding of the bone graft [5]. However, the exact biomechanical properties of dynamic plates remain unknown.

Further development of the dynamic plate model has yielded a unidirectional dynamic plate system that, hypothetically, further increases load sharing properties by allowing the cervical plate to collapse in one direction only as graft subsidence occurs. This study directly compares this plate design to a current FDA approved dynamic plate design, as well as a static plate design, to assist in the understanding of the biomechanical properties of these dynamic plates. 


\section{CHAPTER 2. BACKGROUND}

\subsection{Definitions of Major Concepts}

Subsidence is a term that refers to a height reduction and/or angular distortion due to axial skeleton shortening and deformation. There are several mechanisms of subsidence; loss of vertical height due to aging, disc collapse, and the loss of height at an operative site following surgery on the spine [9]. The loss of vertical height due to aging is multifactoral, essentially involving both the loss of disc height and vertebral body collapse. Surgical subsidence is multifactoral as well. The primary mechanisms are bone resorption and remodeling, and bone graft settling or collapse. Both aging and surgical subsidence tend to cause angular and axial subsidence along the spine, both of which must be addressed [9]. Subsidence can occur in all regions of the spine, but is most evident in the cervical spine [9].

Graft settling (also known as graft collapse) is a process that occurs as bone grafts are placed under load for the first time after surgery, and accounts for an axial bone graft height reduction [9]. The mechanisms of graft settling are numerous, including improper sizing and contouring of the graft material; known to be the primary reason. A properly sized and contoured graft spreads clinical loads over a larger area, thus reducing the load per unit of surface area. Gaps left in the discectomized region contribute directly to the graft height reduction immediately upon being placed under load. The chosen graft material has also been found to have a significant effect on subsidence. Autograft material is considered the "gold standard," while allograft and synthetic materials tend to lack the material properties that allow for proper graft response when placed under load [9]. The choice between cancellous or cortical bone is important. Cortical bone provides the early structural support needed for clinical loads, but due to its density tends to resist vascular ingrowth and the influx of osteoblasts. Cancellous bone lacks the strength of cortical bone, but has favorable osteoconductive properties, and demonstrates lower rates of pseudoarthrosis [9].

Though sometimes perceived as an undesired aspect of cervical spine surgery, graft settling and subsidence are both normal parts of cervical fusion surgery. A natural, measurable amount of graft settling should be experienced as the graft and vertebral endplates are pushed together under clinical loads that are associated with proper arthrodesis formation. As well, a natural measurable amount of subsidence should be expected during bone graft resorption and remodeling.

Stress shielding can occur after anterior cervical fusion surgery with plating systems at the fusion site, due to subsidence and graft settling. After anterior cervical fusion surgery, axial loading on the fusion site is shared between the graft and the instrumentation. Stress shielding occurs after a graft height reduction such that the majority of clinical axial loading shifts from the graft to the instrumentation, causing improper load sharing between the instrumentation and the bone graft [5]. Wolff's law states that as loading on a particular bone increases or decreases, the bone will remodel itself over time to become stronger to resist larger loading, or weaker to resist lesser loads due to turnover as it is less metabolically costly to maintain bone mass [10]. It is theorized that by Wolff's law, reducing the loads placed on the graft via stress shielding 
greatly reduces bone remodeling during fusion, thus greatly increasing the risk of pseudoarthrosis.

\subsection{Dynamic Plate Design}

The need for dynamic instrumentation became evident through years of clinical complications with rigid plate designs including screw backout, screw breakage, construct pullout, suboptimal fusion strength, and fusion failure (pseudoarthrosis) [9]. Numerous generations of rigid plate design attempted to address these issues, but the underlying problem of stress shielding of the union site was not fully understood. Recently, further understanding of the implications of Wolff's law to spinal fusion surgery has resulted in the development of dynamic plate designs. The concept of dynamism has been used in other orthopedic applications, particularly the hip and long bone fractures, and even had been an indirect feature of the first generation of anterior cervical plating systems.

\subsection{Literature Review}

Though anterior cervical plates have been well studied, dynamic plates have only recently been evaluated in ways that include graft height reduction mechanics. Anterior cervical plating was first studied as a surgical technique by Bohler in 1964, who developed the first ventral cervical plate [3]. These plates were further developed in the early 1980s with the Caspar (Aesculap) and Orozco (Synthes) plate designs that were indirectly dynamic in nature, due to the lack of plate-screw fixation. However, the toggling screws caused a lever moment arm that caused a high incidence of screw pullout, and the lack of a screw locking mechanism caused a high incidence of screw backout [3].

The next generation of anterior cervical plates designed in the 1980s was the CSLP (Synthes) and Orion (Sofamor Danek) plates. These plates both addressed incidence of screw backout and pullout with use of fixed angle (non toggling) screws and locking features. However they were not dynamic in any fashion, and experienced much higher fusion failure rates and pseudoarthrosis, theoretically due to stress shielding of the bone graft, discussed in section 2.1.

Since then, several studies on the effects of dynamism in cervical fixation have displayed a reduced incidence of fusion failure with dynamic plate fixation. [3, 14, 15] Stulik et al also saw a significantly reduced incidence of implant complications using dynamic plates [14].

Since the discovery of the effects of dynamism on cervical plate fixation, numerous designs have been approved for human use, but all can be classified as rotationally dynamic, translationally dynamic, or a combination of both. Rotationally dynamic plates typically allow angular translation between the plate and screw, allowing the screw to "toggle" in the platescrew interface. Translationally dynamic plates are not allowed to pivot, but rather typically allow the screws to translate axially, typically by sliding along a rail. 
The most popular plate designs involve allowing both rotational dynamism as well as translational dynamism in limited capacities. Although history has proven that rotationally dynamic plates have a higher incidence of screw pullout, they are desirable from a surgical standpoint because they allow the surgeon a high amount of variability in trajectory during screw placement, which is sometimes necessary in trauma cases. Rotationally dynamic plate designs have a slight advantage in that they allow a small amount of angular subsidence, which has sometimes proven necessary in limited amounts due to uneven graft subsidence [3].

Research on dynamic anterior cervical plating has been limited, and has focused on load sharing interactions from subsidence and graft settling with the use of dynamic anterior cervical plates, as well as optimizing levels and types of plate dynamism. Brodke et al. recently studied the effects of a $10 \%$ subsidence height reduction on a full length interbody spacer placed in a cadaveric specimen on load sharing properties, and found that both rotationally dynamic plates and translationally dynamic plates showed far better load sharing properties than static plates after subsidence had occurred [5]. Advantages of the use of plates that are both rotationally and translationally dynamic were shown by Apjlbaum et al. who performed a recent clinical study on the rotationally and translationally dynamic ABC plate (Aesculap) and found six month fusion rates of $86 \%$ with its use, while still allowing the advantages of toggling screws during spinal fusion surgery [15]. However little research has been done involving the use of limiting dynamism to a single direction (Unidirectional). 


\section{CHAPTER 3. MATERIALS AND METHODS}

\subsection{Device Design}

The cervical plates used for this study were designed and manufactured by Medtronic, Inc. and were customizable to simulate three different plated conditions after installation: static, dynamic-unidirectional, and dynamic-bidirectional. The plate was fully bidirectional without modification, allowing up to $2 \mathrm{~mm}$ axial travel distance between each vertebral fixation. A ratcheting mechanism on the plate was activated to simulate the unidirectional condition that ratcheted every $1 \mathrm{~mm}$ during plate compression. Rigid motion restriction spacers were placed in the plate to simulate the static condition, with the plate fully extended.

\subsection{Specimen Preparation}

Six fresh, frozen human cadaveric cervical (C1-T2) spines were procured from the Medical Education Research Institute (Memphis, TN). The spines were harvested, doublewrapped in plastic bags and stored at $-20^{\circ} \mathrm{C}$ until specimen preparation for preservation of mechanical properties [11]. All spines were screened with anteroposterior and lateral radiographs to exclude any specimens with gross osteopenia or anatomic abnormality, and to verify tissue quality. Radiographs were taken at the Medical Education Research Institute (Memphis, TN) using a digital fluoroscopy machine, as shown in Figure 3.1. The cause of death of each cadaver was unrelated to the cervical spine. The average age for the specimens was $61+/-8$ years. Table 3.1 shows specific specimen information.

The surrounding paravertebral soft tissues were then dissected removing musculature, with care taken to preserve the spinal ligaments, discs, and bone. Excess muscle and adipose tissue were removed from either side of the spinal process. A small portion of the base of each process was then scraped clean of tissue in preparation of target mounting. The $\mathrm{C} 1$ and $\mathrm{T} 2$ levels were then removed. To remove $\mathrm{C}$, an incision was made between the cartilage pads and around the posterior lamina. The $\mathrm{C} 1$ vertebra was then removed by severing the odontoid process and remaining ligament structure around the odontoid process using a small saw. The T2 level was removed in a similar fashion, first making an incision between the processes of $\mathrm{T} 1$ and $\mathrm{T} 2$, and then carefully severing the ligament structure, and zygapophysical capsules.

In order to mechanically test the specimens within the test frame in an upright neutral position, the ends of each specimen were fixated to mechanically compatible endplates with a low melting point bismuth alloy potting kit. Two or three 2-cm long wood screws were driven into the body and spinal arches of the $\mathrm{C} 2$ and $\mathrm{T} 1$ vertebral bodies, as shown in Figure 3.2 (A). The specimen was then placed in an alignment jig and the $\mathrm{C} 2$ or $\mathrm{T} 1$ vertebras were then placed inside a cylindrical mold. Alignment screws were used for correct positioning, and then the low melting point bismuth alloy (Small Parts, Miami Lakes, FL) was poured into the mold, fixating to the screws and vertebral body as shown in Figure 3.2 (B). Once the alloy was left to cool, the 


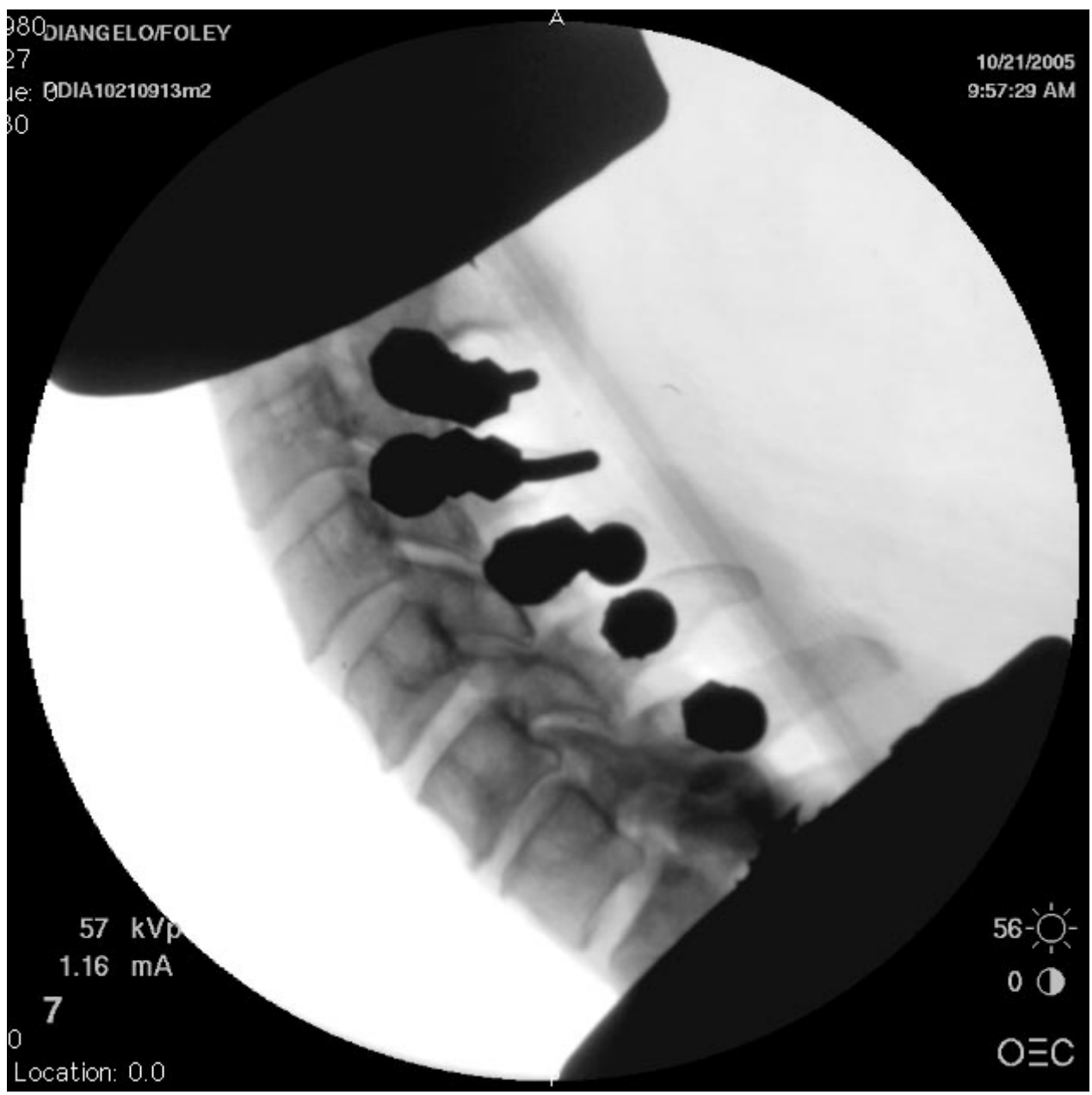

Figure 3.1 Specimen Evaluation. Radiographs such as the one above were taken and evaluated for specimen quality. These images are of specimen IU after the potting and rodding procedure. 
Table 3.1 Specimen Data.

\begin{tabular}{cccc}
\hline Specimen & Age & Sex & Race \\
\hline IX & 57 & $\mathrm{~F}$ & Caucasian \\
IW & 48 & $\mathrm{~F}$ & Caucasian \\
IV & 67 & $\mathrm{M}$ & Caucasian \\
IU & 62 & $\mathrm{M}$ & Caucasian \\
IT & 66 & $\mathrm{M}$ & Caucasian \\
IS & 67 & $\mathrm{M}$ & Caucasian \\
& & & \\
Average & 61.2 & & \\
Max & 67.0 & & \\
Min & 48.0 & & \\
St Dev & 7.5 & & \\
\hline
\end{tabular}




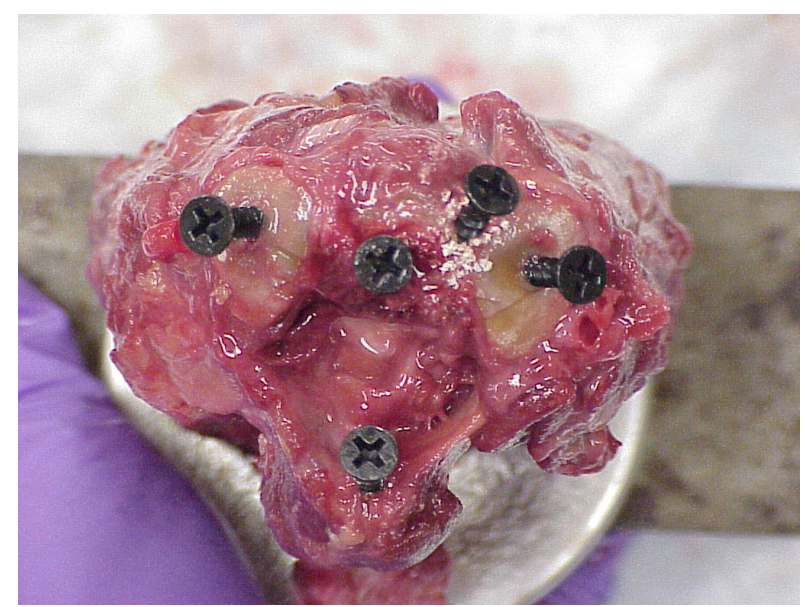

A

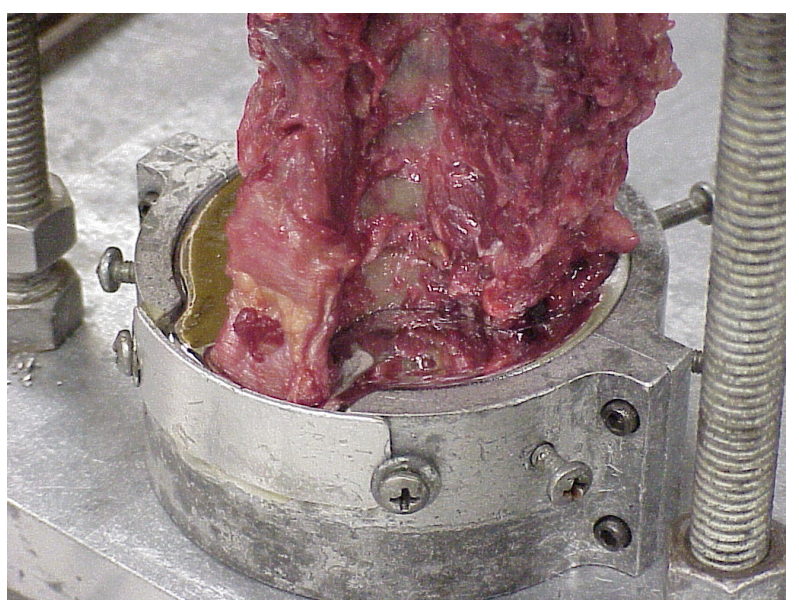

B

Figure 3.2 Spine Potting Procedure. (A) Multiple screws are driven into the endplate and spinal arch of $\mathrm{C} 2$ and T1. (B) The endplate is then placed in a mold and alignment jig, where a low melting point bismuth alloy was poured and fixated to the vertebral body. 
opposite end was fixated in a similar manner. The specimens were then soaked with saline solution, wrapped in cloth, and refrozen at $-20^{\circ} \mathrm{C}$.

To track the motion of each vertebral body, hardware was then fixated to the spinal process of each vertebrae. Size 8/32 threaded rods were driven through the lateral aspects of the spinal process and locked to the spine using nuts and washers. The ends of the threaded rods were then adjusted to permit attachment of light-emitting diode (LED) target arrays used with a motion tracking system, described later. The motion tracking hardware did not interfere with spinal instrumentation placement or natural spine motion.

\subsection{Spinal Conditions}

During surgery, each specimen underwent two single-level discectomies at the C4-C5 and $\mathrm{C} 5-\mathrm{C} 6$ junctions. Two compliant force sensing grafts were then fitted and implanted into the discectomized regions, simulating bone graft material. The customizable cervical plate was then installed per manufacturer specifications. The plate was installed in the static condition, and modified into the bidirectional and unidirectional conditions postoperatively.

The first condition was the harvested condition, for motion data normalization, and did not contain graft load data collection. Postoperatively, the second through fourth spinal conditions were the plated conditions, and were tested in the order of bidirectional, unidirectional, and static.

\subsection{Test Equipment}

The spines were mounted in a programmable testing apparatus and non-destructively tested in the four different spine conditions. The single degree of freedom controlled testing apparatus called a Single Actuator Adaptable Programmable Testing Apparatus (SAAPTA) was used for this purpose, shown in Figure 3.3. The device uses a servo motor load actuator (International Device Corp., Novato, CA) connected to a robotic motor controller (Adept Inc., San Jose, CA) for displacement control. The apparatus is highly adaptable, in that with slight tooling changes, it can be made to test for both axial torsion testing and for bending moment testing.

For bending moment testing, a single axis load cell (Transducer Technologies, Temecula, CA) inline with the force actuator allowed for force data collection and simultaneous moment calculation. Degrees of rotation were collected both by a rotational displacement transducer (Data Instruments, Acton, MA) located at the pin joint between the end of the actuator and loading rod, and a linear transducer (Data Instruments, Action, MA) offset from the loading rod. The loading rod was rigidly coupled to the specimen on one end, and to the force actuator via a pin joint on the other. This connection allowed the actuator to transfer a torsion moment to the specimen, as shown in Figure 3.3. For bending moment testing, specimens were positioned at a $200 \mathrm{~mm}$ offset distance from the actuator loading axis, measured directly from the loading rod. Axial rotation of the test specimens was unconstrained during lateral bending testing, but 


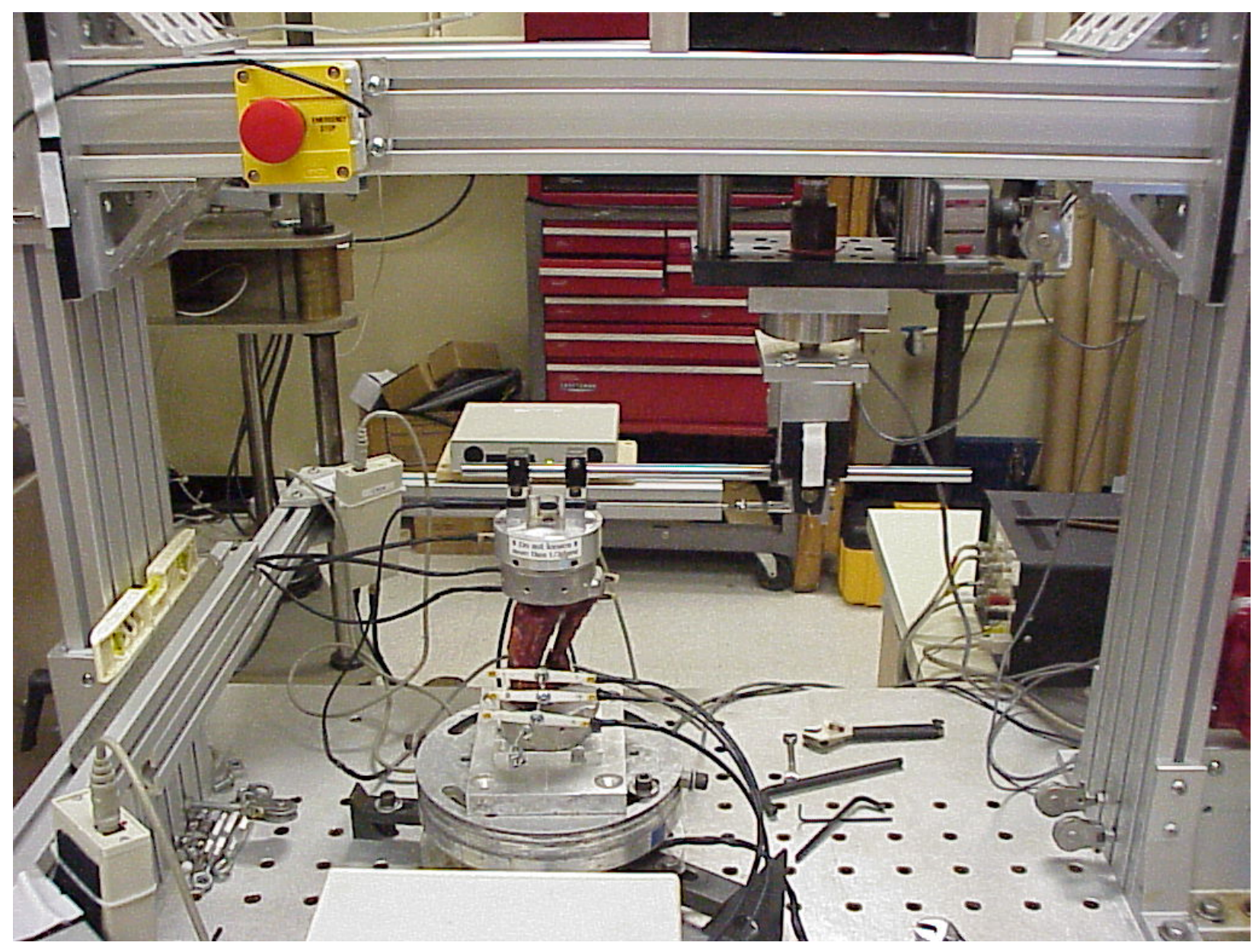

Figure 3.3 Single Actuator Adaptable Programmable Testing Apparatus (SAAPTA). Test frame in a bending moment test. Non-contact motion tracking system is shown attached to the specimen being tested. 
restricted during flexion and extension testing. For lateral loading testing, axial rotation was unconstrained.

For axial rotation, the cervical specimen was placed in an upright position, and the linear actuator provided axial rotational torque to the specimen via a pulley system connected to the bottom of the specimen. A torque load sensor (Transducer Techniques, Temecula, CA) rigidly coupled to the top of the spine provided torsion measurement during axial testing. The torsion sensor was subsequently coupled to the actuator with a linear bearing that provided rotational rigidity, but axial freedom of movement for the actuator, and specimen.

Light-emitting diode (LED) target arrays were rigidly attached to threaded rods passed through the lateral spinal process of each vertebrae, as discussed in section 3.2. A threedimensional non-contact ultraviolet camera tracking system (Origin Instruments, Grand Prairie, TX) was then used to track the individual motion segment units in real time. Both can be seen with Figure 3.3.

To simulate graft height material reduction, while allowing for accurate graft force data collection, a single axis load cell with a load range of 0-200 +/- 5 Newtons (Cooper Instruments, Warrenton, VA) was combined with a compliant device that allowed 0-2 mm of elastic compliance through the 0-200 Newton range. The elastic compliant device was composed of a neoprene rubber (40 DURO) stopper combined with radial plating for proper force distribution. Load cells were each attached to an amplifier unit (Cooper Instruments, Warrenton, VA), and fed into an analogue-digital (A/D) conversion board, as shown in Figure 3.4. Data was then collected via LabVIEW (National Instruments, Austin, TX) and processed in a Microsoft Excel spreadsheet.

\subsection{Non-Destructive Testing Protocol}

All biomechanical testing was performed in the Joint Implant Biomechanics Laboratory in the Department of Biomedical Engineering and Imaging, at the University of Tennessee Health Science Center (UTHSC) in Memphis, Tennessee. The testing protocol followed for this study is that described by DiAngelo et al [8], and is an established tissue testing protocol. All specimens and conditions were tested in flexion, extension, left and right lateral bending, and in left and right axial rotation. Examples of the testing modes are shown in Figure 3.5. During bending motion, the spine was placed in an inverted position in accordance with DiAngelo et al [8], shown in Figure 3.5, and described in Figure 3.6. The inverted position was necessary to produce a moment distribution that generates an in-vivo like motion distribution across the spine, as proven by DiAngelo et al. Prior to testing, each specimen was placed in the test frame in a neutral vertical position in a no load condition.

Bending moment testing was performed under displacement control with a programmed triangular-shaped displacement-time waveform output of $6.4 \mathrm{~mm} / \mathrm{sec}$ for the linear actuator, which corresponds to approximately 2.0 degrees/sec of global spinal rotation. All tests proceeded until either an end loading limit of $3.0 \mathrm{Nm}$ or global rotation of 30 degrees was 


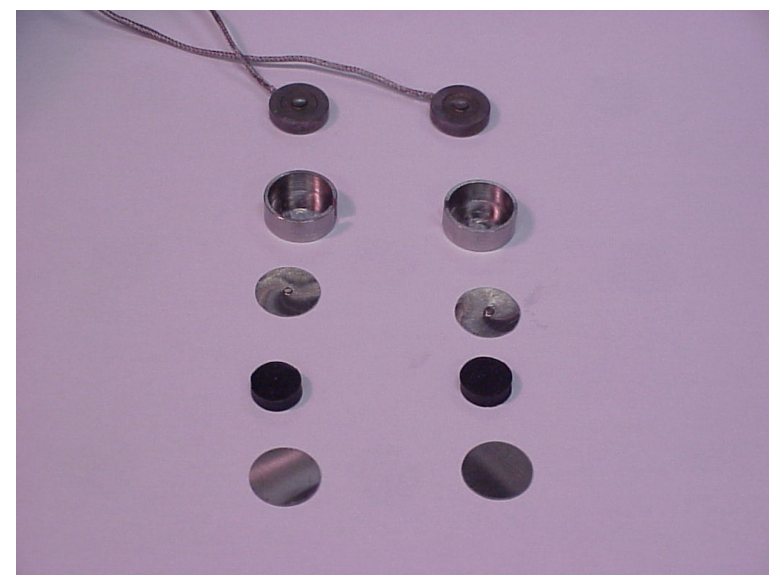

A

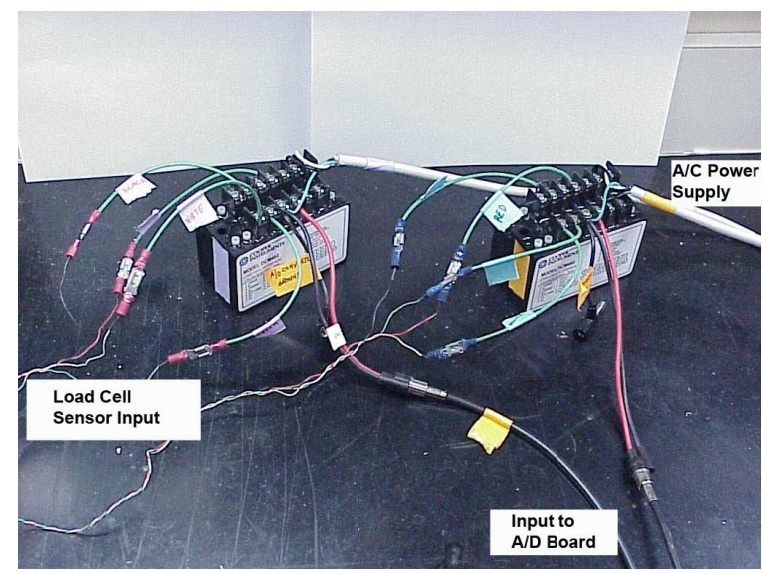

C

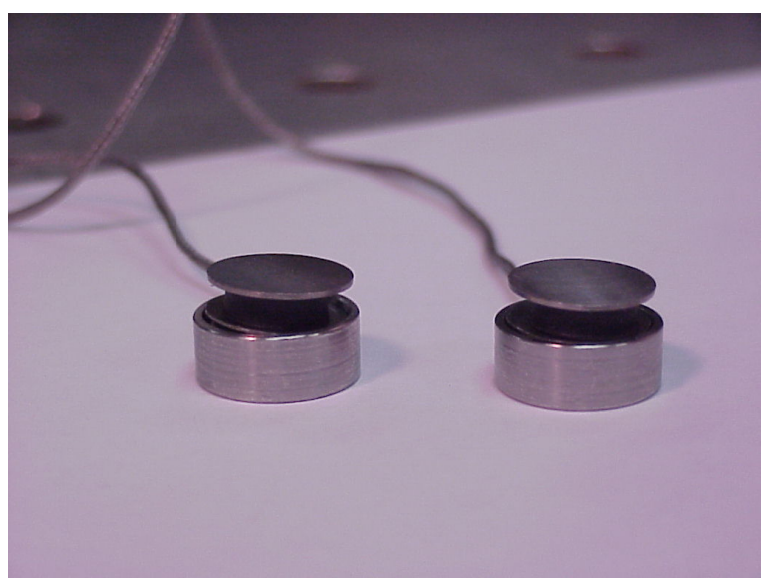

B

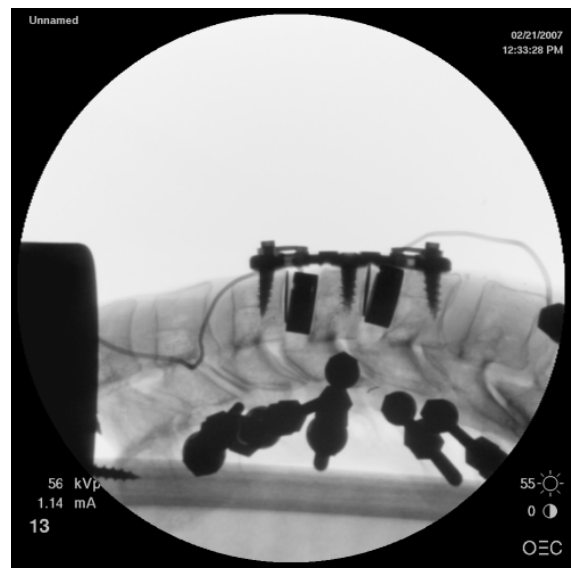

D

Figure 3.4 Compliant Force Sensing Grafts. (A) The compliant force Sensing grafts consisted of load cells that fit into load cups, followed by insertion of a neoprene rubber stopper between load distribution plates. (B) A combined force sensing graft ready for implantation. (C) Amplifier units attaching load cells to the A/D board. (D) Fluoroscope imaging verifying proper load cell and plate placement. 


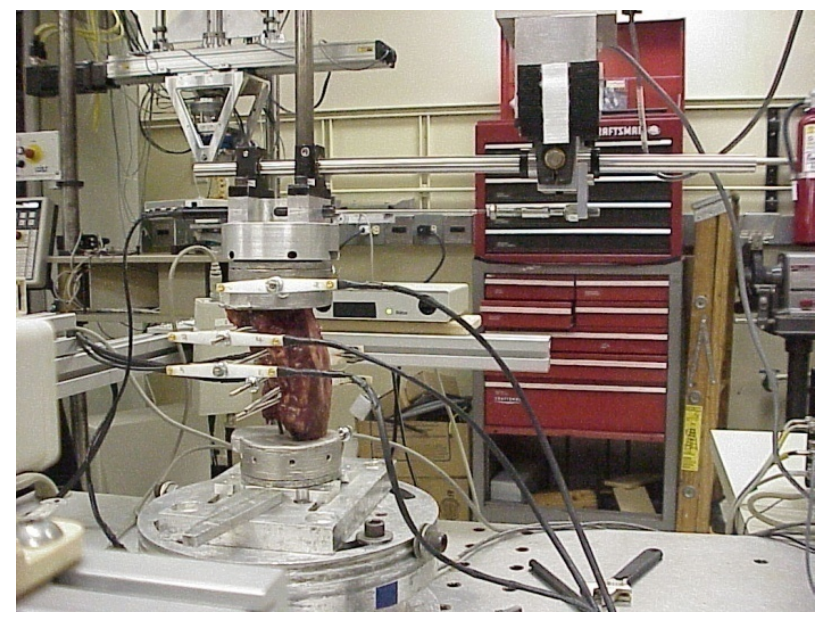

A

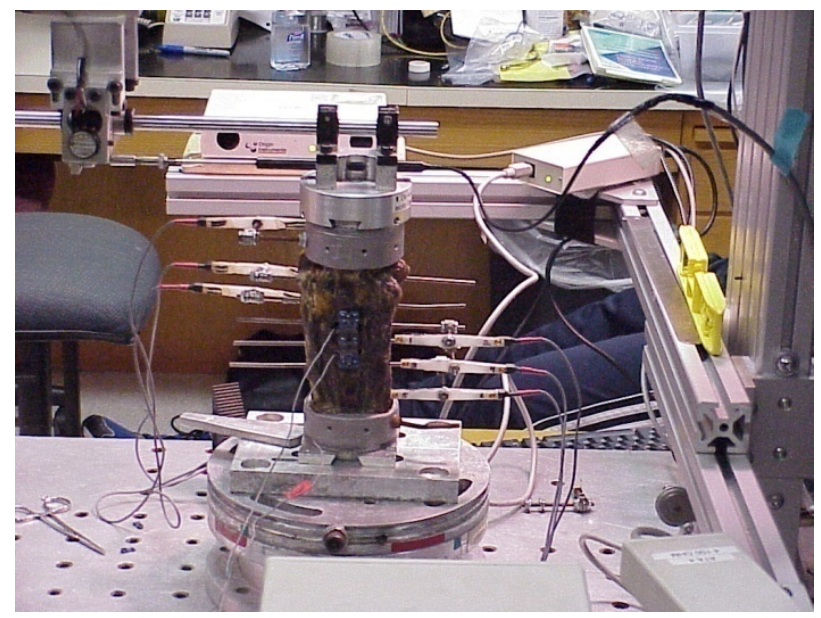

C

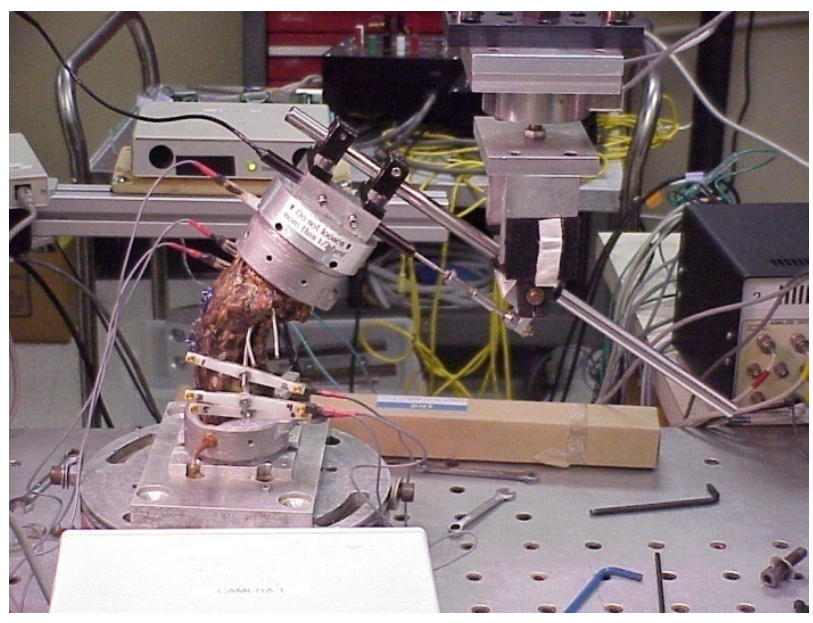

B

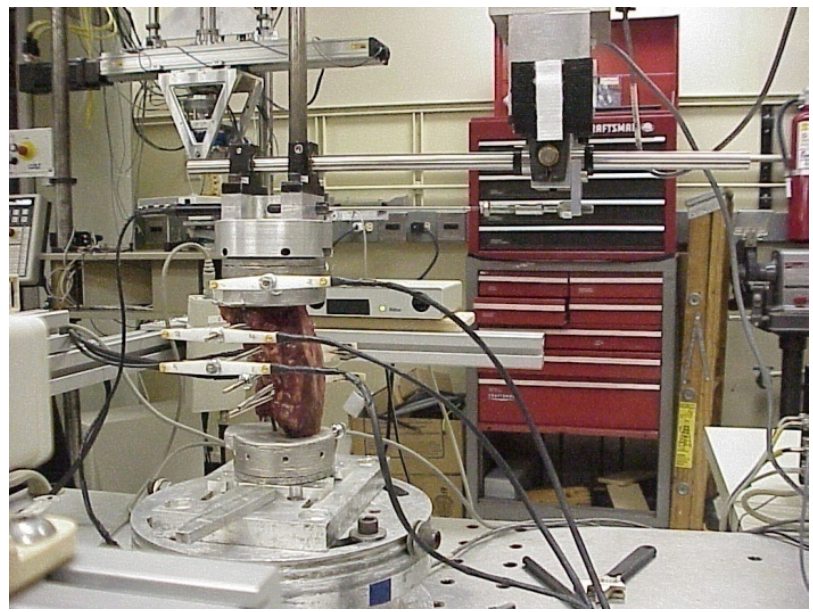

D

Figure 3.5 Test Conditions. (A) Flexion (B) Extension (C) Lateral bending (D) Axial rotation. 


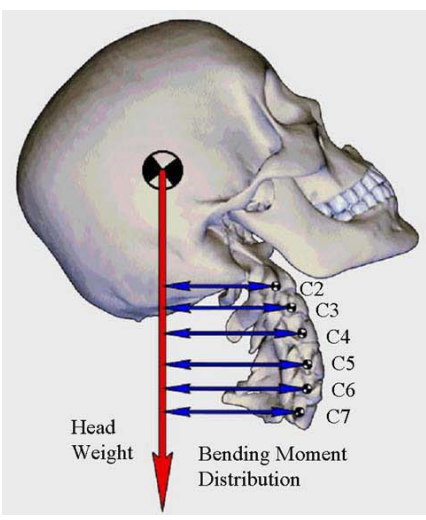

A

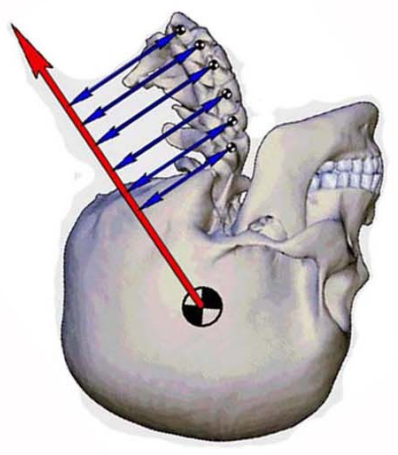

B

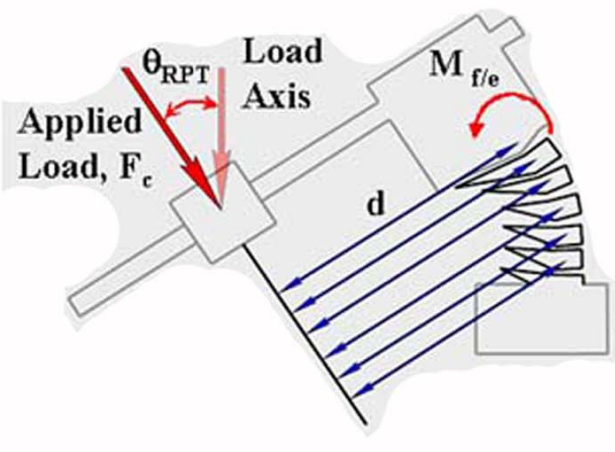

$\mathrm{D}$

Figure 3.6 Sagittal Loading Conditions. (A) Typical head weight acting through the center of gravity produces a force noted by the large arrow which produces bending moments across the vertebral bodies, as denoted by smaller arrows. (B) Inversion of the spine, while simulating the weight of the head, produces similar bending moment distributions at each vertebral body. (C) Reproducing the resultant force due to the weight of head on a linear bearing reproduces an invivo-like moment distribution. [Adapted with permission. Foley KT, DiAngelo DJ, Rampersaud YR, et al. The in vitro effects of instrumentation on multilevel vertical strut-graft mechanics. Spine 1999;24:2366-2376.] 
reached. The spines were preconditioned with five cycles before formal testing. Each formal test included three loading cycles. To avoid tissue dehydration, the spines were moistened regularly throughout the study with $0.9 \%$ saline solution.

\subsection{Data Management and Statistical Processing}

Measurements included rotational motion of individual vertebral bodies, global spinal rotation, and loads in flexion bending, extension bending, left and right lateral bending, and in left and right axial rotation. During the three plated conditions, graft loading data sets were collected from the compliant force sensing grafts. Variations in measurements were analyzed and compared at a common global moment goal of $3 \mathrm{Nm}$ for all spines.

Global rotation and applied load data were used to calculate overall spinal stiffness and flexibility. All instrumented specimens were normalized to their respective harvested data before comparison to account for intrinsic differences in specimen tissue.

Motion response was evaluated by comparing the relative contribution of the C4-C6 levels to the overall global rotation of the spine, then normalizing to harvested data. As well, combined flexion + extension bending data tables, left + right lateral data tables, and left + right axial rotation tables were created, to analyze the full range of motion for each movement plane.

Motion segment unit (MSU) percent contribution was also calculated from motion data. MSU percent contribution is calculated by comparing each MSU spinal level to overall spine motion, expressed as a percent contribution. Percent contribution is especially useful analyzing individual level motion involvement, and adjacent MSU involvement. Similar to normalized motion, combined tables were formed for the full ranges of motion of flexion + extension, left + right lateral bending, and left + right axial rotation.

For statistical analysis of motion data, a one-way repeated measures analysis of variance (ANOVA)was performed, followed by a Holm-Sidak multiple comparison test using the harvested data as a control, with significance level $\alpha=0.05$. If all plated conditions were significantly different than harvested, harvested was removed from the analysis. A Student Newman Kuels (SNK) multiple comparison test was then performed on the remaining conditions, with a significance level $\alpha=0.05$.

For the force sensing graft load data, data points were analyzed by peak load, preload, overall load change, and preload change. Typical loading curves with labeled parameters are shown in Figure 3.7.

During the analysis, graft loading data sets were analyzed for the individual C4-C5 and C5-C6 instrumented levels, and then a combined superior + inferior analysis was performed, accounting for all levels by spine condition. 


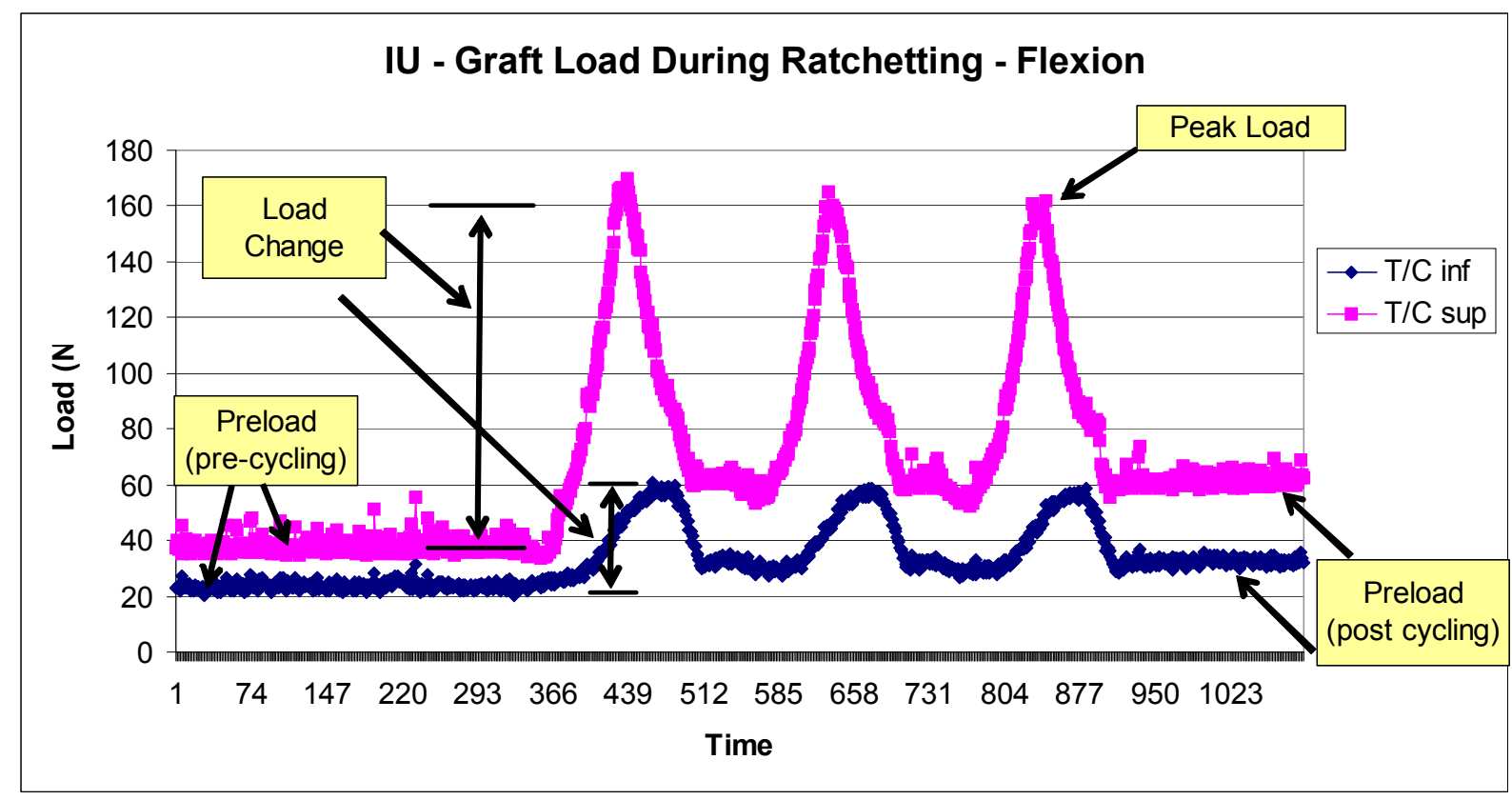

Figure 3.7 Typical Graft Load Cell Data Plot. Specimen IU during flexion testing of the unidirectional plate. "T/C inf" is the inferior C5-C6 level graft load cell, and "T/C sup" is the superior C4-C5 level graft load cell. Preload (Pre and Post cycling), Peak Load, and Load Change are all indicated. The Preload shift (not indicated) demonstrates the effect of the unidirectional feature, and its effect on the preload. 
For force sensing graft load data, a one-way repeated measures Analysis of Variance (ANOVA) followed by a Student Newman Kuels (SNK) multiple comparison test was performed comparing the three plated conditions, with a significance level $\alpha=0.05$. 


\section{CHAPTER 4. RESULTS}

\subsection{Recorded Levels of Graft Collapse}

Recorded levels of collapsing for the unidirectional plates during flexion are summarized in Table 4.1 below. Flexion is the only motion that caused unidirectional plate collapsing. The inferior C5-C6 level's average rate of reduction $(1.2 \mathrm{~mm})$ was higher than the superior C4-C5 level, and a statistical t-test yields $\mathrm{P}=0.076$.

\subsection{Normalized Flexibility}

The mean flexibility values for the three different spine conditions for each test condition are summarized in appendix A, along with standard deviations and statistically significant differences. Figure 4.1 shows global normalized flexibility values for flexion and extension testing of the three instrumented plates. The dynamic plates were significantly different from each other was in extension only, where the unidirectional plate was less flexible than the bidirectional plate. Both dynamic plates were significantly different from the static plate in flexion and left lateral bending, but only the unidirectional plate was significantly different from the static plate in extension. All plated conditions were significantly different from harvested in flexion and right lateral bending, and only the static plate was significantly different from harvested in extension.

\subsection{Normalized Motion}

Normalized motion at the operated levels for the three plated test conditions are summarized in Appendix B, along with standard deviations and statistically significant differences. An example of normalized motion is shown in Figure 4.2, showing combined left and right lateral bending results of the three instrumented plates. The unidirectional plate differed from the bidirectional plate only in combined flexion + extension, where the unidirectional showed less motion than the bidirectional. Both dynamic plates were significantly different from the static condition in left and right lateral bending, and in left and right axial rotation. Only the bidirectional plate was significantly different from the static in combined flexion + extension. All instrumented conditions were significantly different from the harvested spine condition in flexion, extension, and both left and right lateral bending. Only the static plate was significantly different from the harvested spinal condition in left and right axial rotation.

\subsection{Motion Segment Unit Percent Contribution}

Motion segment unit (MSU) percent contribution data for the three different test conditions are summarized in Appendix C, along with standard deviations and statistically significant differences. An example of MSU percent contribution data is presented in Figure 4.3, for flexion and extension. Motion transfer is particularly evident in these figures between the C5- 
Table 4.1 Observed Unidirectional Plate Collapse. The unidirectional plate only reduced in length during flexion testing.

\begin{tabular}{lcc}
\hline \multicolumn{3}{c}{ Observed Ratchetting during Flexion $(\mathbf{m m})$} \\
\hline Specimen & Inferior & Superior \\
\hline IS & 1 & 1 \\
IT & 2 & 1 \\
IV & 1 & 0 \\
IW & 1 & 1 \\
IX & 1 & 0 \\
IU & 1 & 1 \\
Mean & 1.2 & 0.7 \\
\hline
\end{tabular}



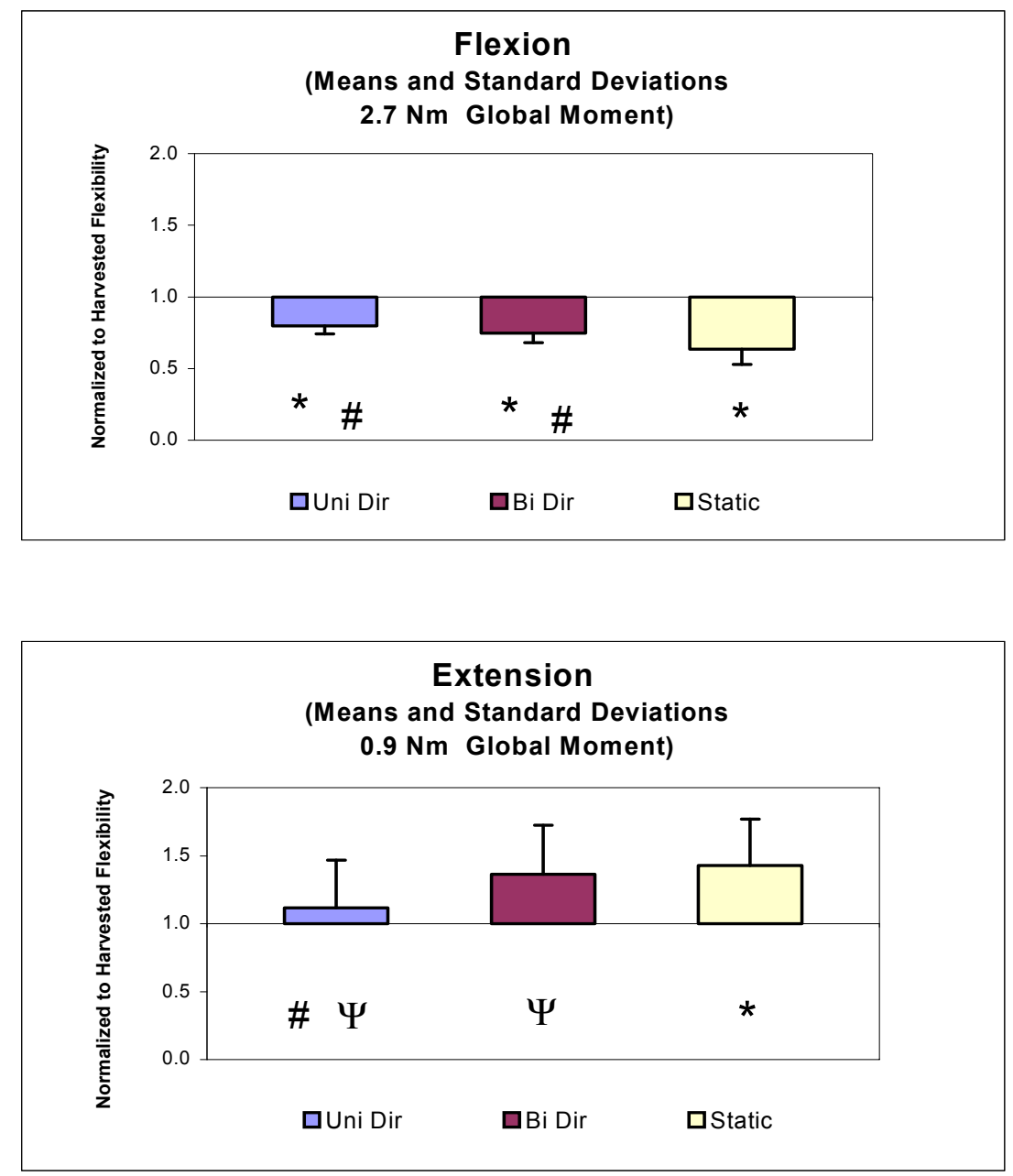

Figure 4.1 Normalized Flexibility for Flexion and Extension. All values are normalized to the harvested spine condition, with statistical differences noted. (* Signifies significant difference from the harvested condition. \# Signifies significant difference from the static condition. $\Psi$ Signifies significant difference between the dynamic conditions.) 


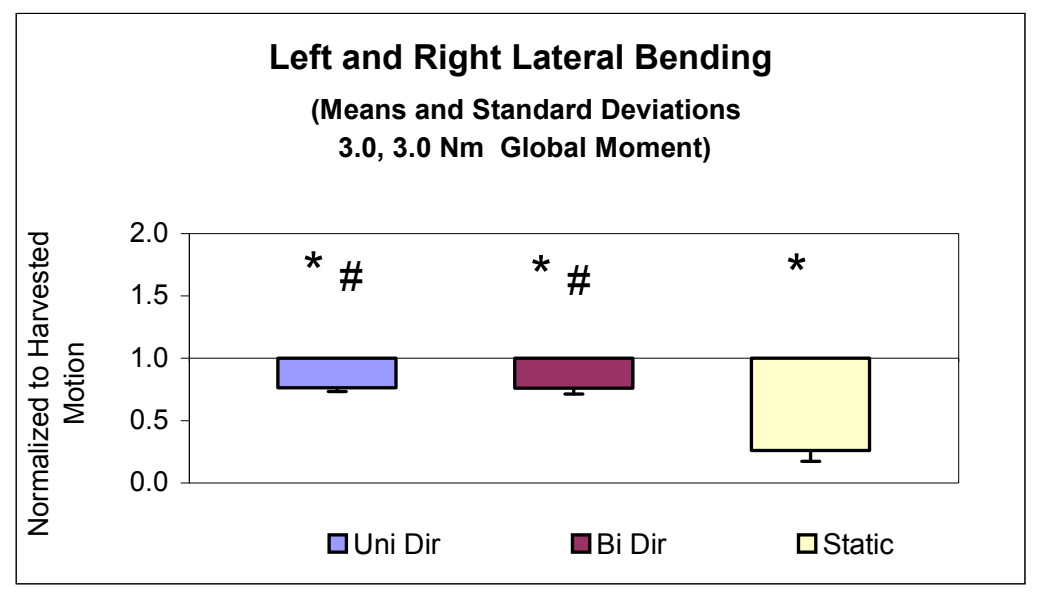

Figure 4.2 Normalized Motion for Combined Left and Right Lateral Bending. All values are normalized to the harvested spine condition, with statistical differences noted. (* Signifies significant difference from the harvested condition. \# Signifies significant difference from the static condition. $\Psi$ Signifies significant difference between the dynamic conditions.) 

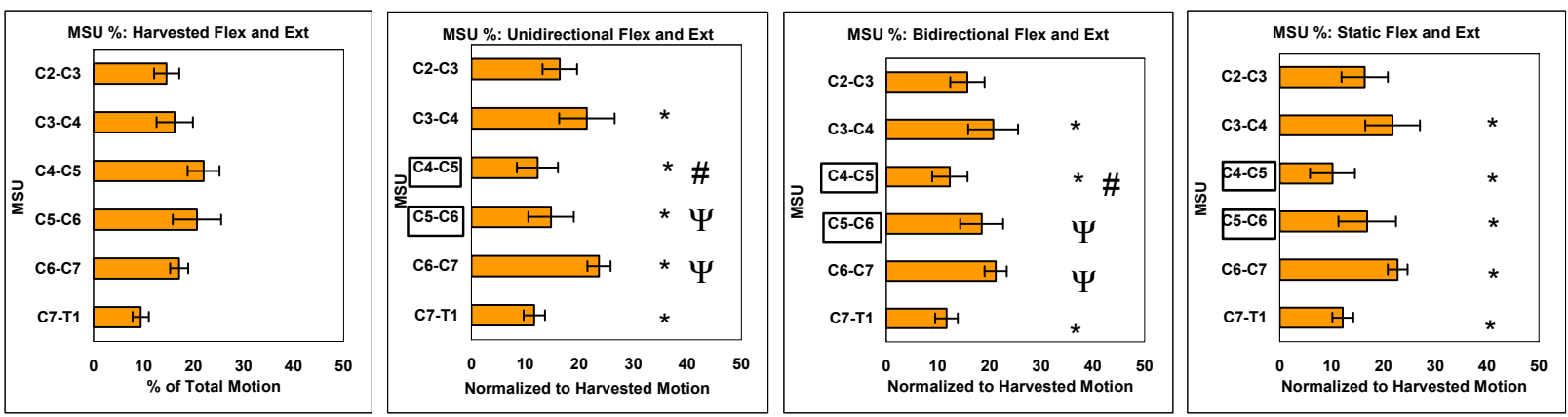

Figure 4.3 Motion Segment Unit (MSU) Percent Contribution for Combined Flexion and Extension. Instrumented levels are indicated by box. An increase or decrease in percentage indicates an increase or decrease in motion. (* Signifies significant difference from the harvested condition. \# Signifies significant difference from the static condition. $\Psi$ Signifies significant difference between the dynamic conditions.) 
C6 and the C6-C7 levels. The only instances where the dynamic plates differed from each other was in the instrumented C5-C6 level and the adjacent C6-C7 level during the extension and combined flexion + extension analysis. In this case, there was a significantly higher amount of motion transfer from $\mathrm{C} 5-\mathrm{C} 6$ to $\mathrm{C} 6-\mathrm{C} 7$ in the unidirectional plate versus the bidirectional plate. The dynamic plates were significantly different from the static condition at the C7-T1 level during flexion, and the instrumented C4-C5 level in combined flexion + extension. Nearly all levels of the dynamic plates were significantly different than the static condition in right lateral bending and combined left + right lateral bending. There were no significant differences between instrumented conditions during axial rotation. In most instances, instrumented conditions were significantly different from the harvested spine condition in flexion, extension, and left and right lateral bending. The only significant differences from harvested axial rotation testing were in the C5-C6 instrumented levels during left axial rotation, and the C2-C3 and C7-T1 levels for instrumented conditions during both left and right axial rotation.

\subsection{Peak Graft Loads}

Peak measured graft load data for the three different test conditions are summarized in Appendix D, along with standard deviations and statistically significant differences. Peak loading is important as vertebral endplate collapse can occur if peak loading is too high. Examples of the peak graft loading data and suggested statistical significance for the combined superior + inferior levels during flexion and extension testing are presented in Figure 4.4. There was no case where there were significant differences between the dynamic plated conditions. The dynamic plated conditions were significantly higher than the static plated condition, particularly in the inferior C5-C6 level in flexion testing, left and right lateral bending, and left and right axial rotation. A trend suggests that the unidirectional plate was significantly higher than the static plate in extension, as discussed in Figure 4.4.

\subsection{Graft Load Change}

Change in measured graft load data are summarized in Appendix E, along with standard deviations and statistically significant differences. Graft load changes are important, as they are an indication of the load transfer dynamics that occur during the bending moments and axial rotation. Figure 4.5 shows flexion and extension bending data for change in graft load, as an example of the data set. There were no significant differences between the dynamic plated conditions. The load change in the dynamic plated conditions were significantly higher than the static plated condition in both levels of flexion testing, and in the inferior and combined superior + inferior grafts for right lateral bending. Only the unidirectional plate was significantly higher than the static plated condition in left lateral bending, for the combined superior + inferior graft load change analysis. There were no significant differences between plated spine conditions in axial rotation or extension. 

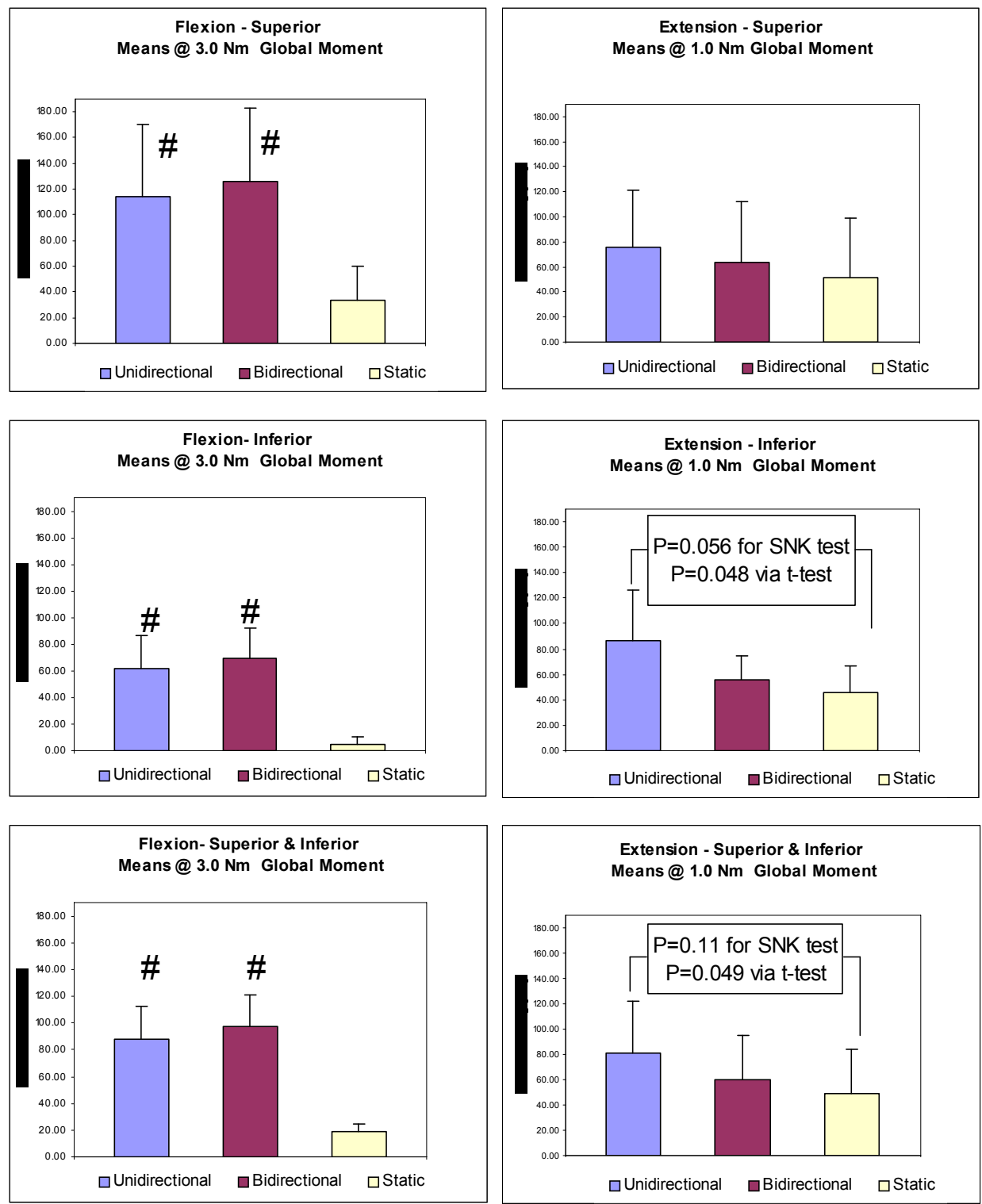

Figure 4.4 Suggested Significance in Peak Graft Loads for Flexion and Extension. P values between the unidirectional plate and static plate during extension testing suggests a trend that the unidirectional plate showed much higher peak loads than the static plate in extension bending. This trend suggests better load sharing capabilities by the unidirectional plate in extension. However, this would have to be verified with a higher specimen study. (\# Signifies significant difference from the static condition. $\Psi$ Signifies significant difference between the dynamic conditions.) 

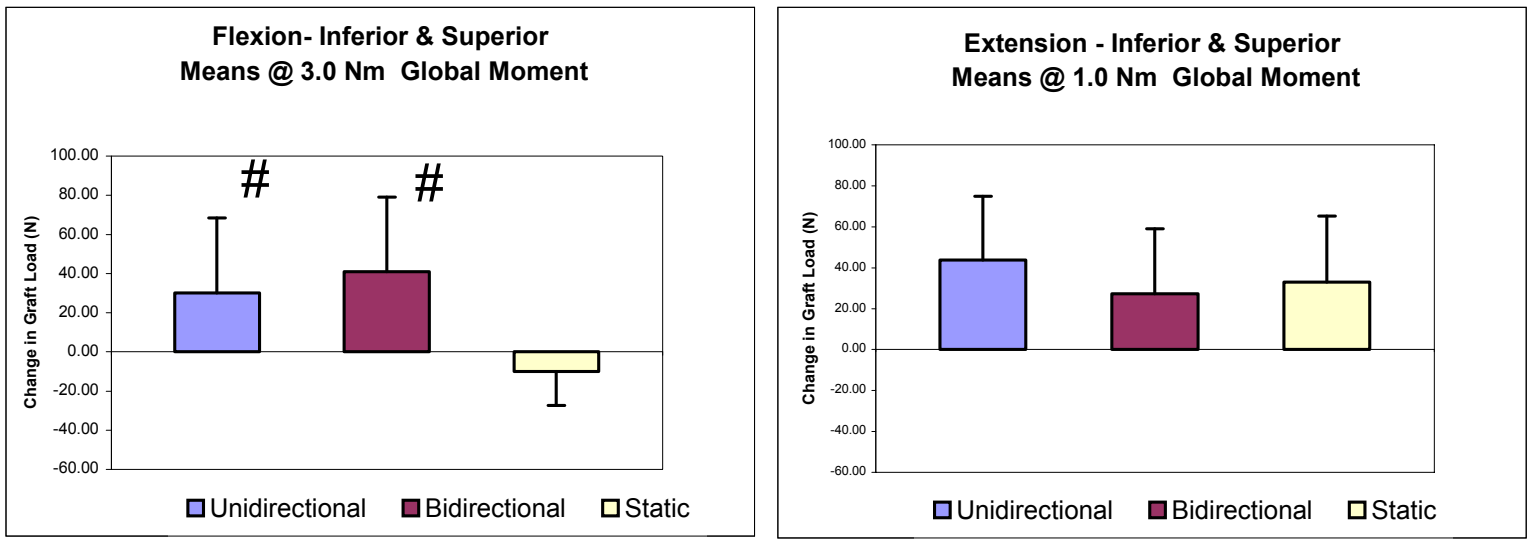

Figure 4.5 Combined Superior and Inferior Change in Graft Loads for Flexion and Extension. Values are combined totals from both the superior C4-C5 and inferior C5-C6 levels. (\# Signifies significant difference from the static condition. $\Psi$ Signifies significant difference between the dynamic conditions.) 


\subsection{Graft Preloads}

Graft preload data sets are summarized in Appendix F, along with standard deviations and statistically significant differences. Graft preloads are important, as they are the baseline loads that the graft sees when not in motion. It is also important to note that there is no motion accounted for in these data sets, and the loads recorded are immediately following the motion cycle testing. An example of graft preload data is demonstrated in Figure 4.6. There were no significant differences between the dynamic plated conditions. However the dynamic plates showed significantly higher preloads than the static plated condition in nearly all cases of the inferior level, and combined superior + inferior analysis. However there were very few significant differences in the superior level, where only flexion and left lateral bending showed higher preloads than the dynamic plates compared to the static plated condition.

\subsection{Graft Preload Change}

Changes in graft preload are summarized in Figure 4.7 along with standard deviations and statistically significant differences. The change in graft preload is important, as it demonstrates any baseline shift that may have occurred in the preload. The only significant difference noted was the unidirectional plate during flexion. In both instrumented levels, the unidirectional plate had a significantly higher preload change during flexion than the bidirectional and static plates. 

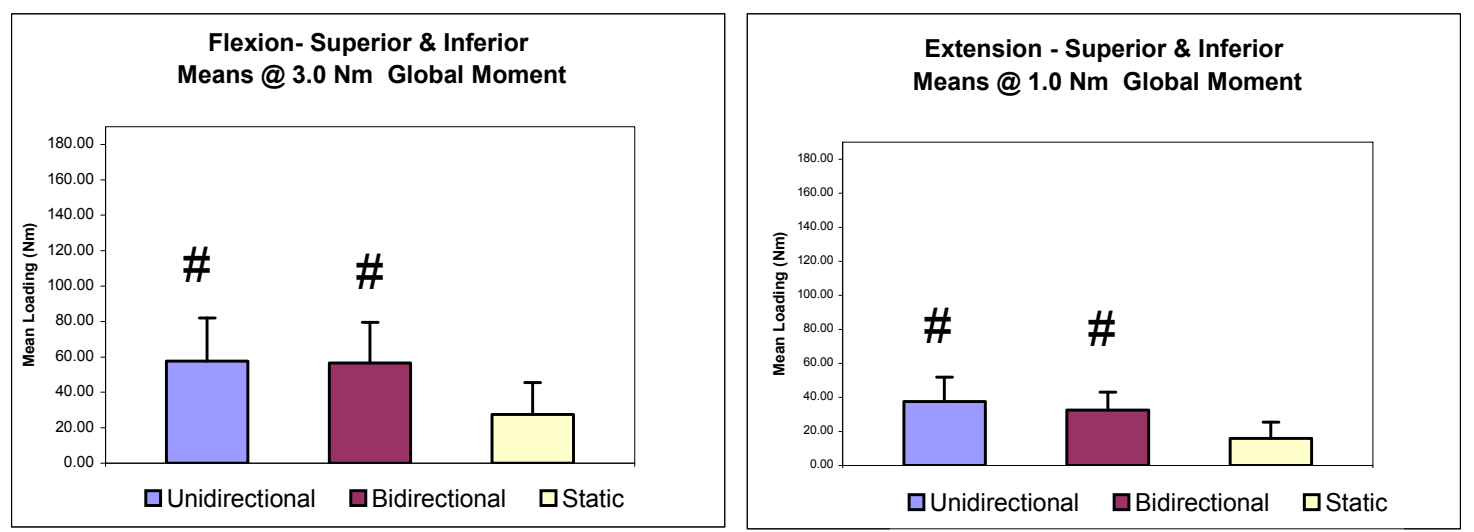

Figure 4.6 Combined Superior and Inferior Graft Preloads for Flexion and Extension. Values are combined totals from both the superior C4-C5 and inferior C5-C6 levels. (\# Signifies significant difference from the static condition. $\Psi$ Signifies significant difference between the dynamic conditions.) 

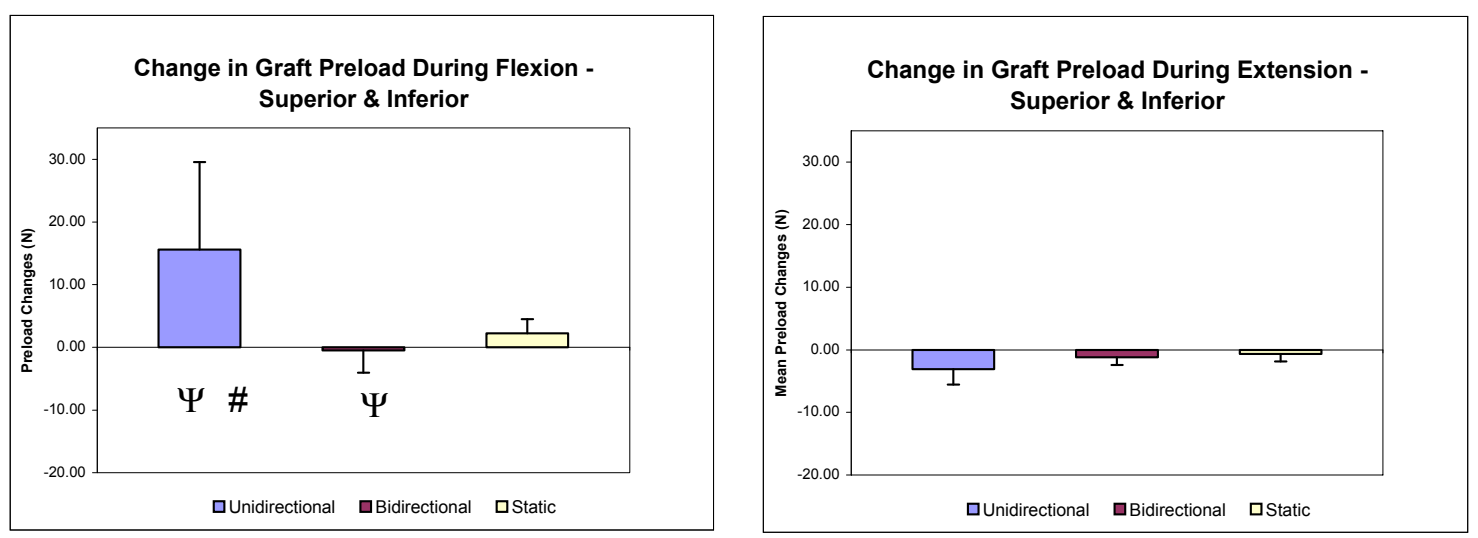

Figure 4.7 Change in Graft Preloads for Flexion and Extension. Values are combined totals from both the superior C4-C5 and inferior C5-C6 levels. (\# Signifies significant difference from the static condition. $\Psi$ Signifies significant difference between the dynamic conditions.) 


\section{CHAPTER 5. DISCUSSION}

\subsection{Inferior versus Superior Level Comparison}

Many comparisons can be made when comparing superior versus inferior involvement. Most of the data suggests more dynamic action at the inferior C5-C6 level than the superior C4$\mathrm{C} 5$ level. When analyzing Table 4.1 , there was a trend $(\mathrm{P}=0.076$ via $\mathrm{t}$-test $)$ that the unidirectional plate reduction was higher at the inferior (C5-C6) region. A statistical power test with alpha $=0.050$ was 0.284 . (A value $<0.8$ indicates we are less likely to detect a difference when one actually existed.) This finding suggests that higher loads are seen in the inferior level, which is consistent with prior studies and kinematic models. Power calculations support that we may have failed to find significance with the current sample size, when one actually existed.

There is very little that can be used to distinguish differences in inferior versus superior involvement from the normalized flexibility and motion data, due to the nature of those analyses. Both combine the fixated levels and compare them to the entire spine, and do not compare the individual levels. However the MSU percent contribution is a valuable tool. For combined flexion + extension rotation, the dynamic plates demonstrated less motion restriction at the $\mathrm{C} 4-$ C5 level than the static plate. However at the C5-C6 level, the unidirectional plate was significantly more motion restrictive than the bidirectional and static plates. Considering that there was more plate reduction at the inferior level, this would lead us to conclude that as the plate collapses, more motion restriction is present in the flexion-extension motion plane. It is also noteworthy that the only significance we saw at the operated levels were at the inferior level.

Large differences between the superior and inferior levels are apparent when analyzing the compliant graft load cell data. Higher peak loads were seen at the superior level during flexion for all plated conditions. This means more load was being transferred into the graft at that level, and could be partially the reason for the trend that less unidirectional plate collapse was seen at the superior level. There were little differences between the superior and inferior loads in all other test conditions.

During the load change analysis, differences between superior and inferior levels are only seen in flexion bending. In the dynamic plates, much higher load changes are seen in the superior level than the inferior. Similar to peak loading, this means more load was being transferred into the graft than the plate at that level, and could partially explain why less unidirectional plate collapse was seen at the superior level. There were little differences between the superior and inferior loads in all other test conditions.

Looking at preloads, there is little difference between the inferior and superior levels, except for axial rotational testing. Graft preloads were far less for the superior level during both left and right axial testing. The cause for this is unknown. 


\subsection{Static versus Dynamic Plate Comparison}

Our findings illustrate that both dynamic plates display far better load sharing properties than the static plate, while generally allowing more motion. During the normalized flexibility analysis in flexion bending testing, both dynamic plates showed more motion than the static plate. This makes sense kinematically, as when the spine moves into flexion, the static plate will resist the motion directly, while both dynamic plates will collapse. As well, during lateral bending, the dynamic plates proved to be much more flexible than the static plate.

In the normalized motion analysis, both dynamic plates demonstrated much more motion at the operated levels than the static plate in lateral bending and in axial rotation. These findings are consistent with past studies of dynamic plates, as well as dynamic plate design theory [9, 14, $16]$.

Using the peak graft loads analysis, we saw significantly higher peak loads in the dynamic plates for all superior + inferior analyses except during extension. For extension, there were no significant differences via ANOVA and SNK analysis. However, a closer look at the peak graft loads shows evidence that if the sample size were higher, the unidirectional plate may demonstrate higher peak loads than the static plates. (See Figure 4.4) We also saw higher overall graft load changes in the dynamic plates in both levels of the flexion analysis, and in the combined superior + inferior analyses of left and right lateral bending. These findings indicate better load sharing characteristics between the plate and the force sensing graft for the dynamic plates. These findings are consistent with past studies of dynamic plates, as well as dynamic plate design theory $[9,14,16]$.

\subsection{Unidirectional versus Bidirectional Plate Comparison}

Comparing the two dynamic plate designs, we generally saw evidence of less motion from the unidirectional plate versus the bidirectional plate, as well as more consistent loading of the grafts from the unidirectional plate.

The normalized flexibility of the bidirectional plate was significantly higher than the unidirectional plate during extension loading. As well, the normalized motion for the unidirectional plate was significantly less than the bidirectional plate for combined flexion plus extension rotation. This was evidence of the increased movement provided by the bidirectional plate design. In extension and combined flexion + extension, there was significantly less contribution at $\mathrm{C} 5-\mathrm{C} 6$ for the unidirectional plate than the bidirectional plate.

During the MSU percent contribution analysis, there was significantly higher transference to the adjacent C6-C7 level shown in flexion + extension for the unidirectional dynamic plate. During lateral bending, there was a significantly less percent contribution of motion at C7-T1 level for the unidirectional plate (15 degrees) versus the bidirectional plate (17 
degrees). This would infer that there might be less percent contribution from the bidirectional plate in lateral bending, but the trend was not seen in any other lateral bending tests.

During the graft load cell analysis of peak graft loads, graft load changes, and graft preloads no direct significant difference was found via repeated measures ANOVA and SNK testing. However, significance is seen specifically when looking at the change in preloads during flexion. The purpose of this test was to determine the effect of the unidirectional plate height reduction on graft loading, and a comparison of that change to the other plates. Once the ratcheting mechanism of the unidirectional plate was activated, a significant increase in the baseline grafts preload occurred during flexion testing for the unidirectional plate compared to the bidirectional and static plates. As is clearly demonstrated, an average preload shift of 15.6 Newtons occurred per operated level in the unidirectional plate. This increased shift in the baseline graft preload for the unidirectional plate remained present during all subsequent tests. This significant baseline load shift theoretically increases resting preload, and reduces the overall load transients placed on the graft, as peak loads and load changes are not significantly higher. Because of this, it can be inferred that the unidirectional plate provides a more "continuous loading" of the graft, as the overall change in graft loads during movement will be less in the unidirectional plate. As such, unidirectional plates may minimize the incidence of pseudoarthrosis by maintaining contact at the graft-endplate interface. (Graft load changes in Appendix E do not take into account the baseline shift that occurs during initial ratcheting, as the unidirectional plate had already been ratcheted before our test.)

\subsection{Study Limitations}

This study was limited by the number of dynamic plate designs tested. There are numerous dynamic cervical plate designs currently approved for use, including toggling screws and plates that allow limited screw/plate translation. This was a study design decision, due to limitations of those particular designs. Toggling screws exhibit high potential for screw pullout due to the tendency to lever the screws as it rotates, inducing axial screw forces above bone fixation strength [16].

Another limitation of the study is that our force sensing grafts only simulate graft settling, and not collapse or true subsidence due to their elastic properties. Designing a compliant graft load cell that only collapsed is feasible. However, design concepts simulating collapse involve resetting the load cell to its uncollapsed state between every test, which would have been detrimental to tissue quality, and would have been noncompliant with the cycling testing of the protocol used. (See preconditioning and loading cycles, described in section 3.5.) Simulation of true subsidence over a long period of time was also not found to be feasible in an in vitro study.

When analyzing motion data, a non-instrumented "harvested" condition is used for normalization. A limitation of the force sensing graft data is that no harvested data was available as a control. Gross implantation of force sensing grafts for the harvested condition would require surgery, which by definition is no longer a "harvested" control state. 
Though using a plate that could simulate multiple plate designs was convenient for this study and tissue preservation, a limitation of the plate design used was that the plate was installed with the plate fully extended in the static condition. This could not be minimized, due to plate design. Theoretically, this could allow little axial extension of the plate when made bidirectionally dynamic. However in practice, all plates visibly collapsed approximately $1 \mathrm{~mm}$ per level when taken from the static to dynamic condition, and axial plate extension was still evident during bidirectional extension and lateral bending tests, regardless of this limitation.

During our testing, steps were made to simulate in vivo conditions as close as possible. However, the study was limited due to the condition of the cadaveric specimens combined with the idealized loading conditions not necessarily being those seen in-vivo. As mentioned, this limitation was minimized as much as possible by protocol and procedural design.

And finally, this study was limited by sample size. With only six specimens it is possible that significance between test conditions were not seen, that would have been with a higher sample size. In some cases, power calculations were made that proved this. 


\section{CHAPTER 6. CONCLUSIONS}

A compliant interbody graft was developed and used to determine the graft load properties of three different anterior cervical plate designs. To our knowledge, this is the first attempt at an in vitro simulation of a real time graft height reduction to study dynamic plating, and may lead to further development and use of this bone height reduction simulation system.

Consistent with other biomechanical studies, both dynamic anterior cervical plates demonstrated load sharing capabilities superior to that of a static plate. Improved load sharing properties limit stress shielding at the graft - endplate interface and help promote bone formation and growth. More plate collapsing (ratcheting) was seen at the inferior (C5-C6) level. Subsequently, more motion conservation was seen at the inferior level. It can be implied that these two findings are directly related. The unidirectional plate had less motion in flexion/extension, and maintained continuous graft loading unlike the bidirectional plate.

The collapsing feature of the unidirectional dynamic plate was proven to function correctly during flexion, significantly increasing the baseline load of all subsequent graft loading. Although the baseline loading was significantly increased in the unidirectional plate, peak graft loads for the unidirectional plate were not significantly higher than the bidirectional plate in any other loading condition.

The unidirectional dynamic plate was found to limit motion and maintain compressive loads on the graft. It is plausible that this more consistent loading feature of the unidirectional plate may lead to fostering better bone growth. This finding supports thinking that unidirectional dynamic plates may lead to improved fusion rates and decreased incidences of pseudoarthrosis. However, these findings need to be supported with animal studies and in vivo clinical studies to confirm these findings. 


\section{LIST OF REFERENCES}

1. Bailey RW, Badgley CE. Stabilization of the cervical spine by anterior fusion. Am J Orthop. 1960; 42-A: 565-594.

2. Smith GW, Robinson RA. The treatment of certain cervical-spine disorders by anterior removal of the intervertebral disc and interbody fusion. J Bone Joint Surg Am. 1958; 40: 607-624.

3. Bohler J, Gaudernak T. Anterior plate stabilization for fracture-dislocations of the lower cervical spine. J Trauma. 20(3):203-205, 1980.

4. Foley KT, DiAngelo DJ, Rampersaud YR, Vossel KA, and Jansen TH. The in vitro effects of instrumentation on multilevel cervical strut-graft mechanics. Spine. 1999;24:2366-2376.

5. Brodke DS, Klimo P Jr., et al. Anterior cervical fixation: Analysis of load sharing and stability with use of static and dynamic plates. J Bone Joint Surg Am. 2006; 88: 1566-1573.

6. DiAngelo DJ, Foley KT, Vossel KA, Rampersaud YR, and Jansen TH. Anterior cervical plating reverses load transfer through multilevel strut grafts. Spine. 2000;25:783-795.

7. Tye GE, Graham RS, Broaddus WC, Young HF. Graft subsidence after instrument-assisted anterior cervical fusion. J Neurosurg. 2002;97(2 Suppl):186-192.

8. DiAngelo DJ and Foley KT. An improved biomechanical testing protocol for evaluating spinal arthroplasty and motion preservation devices in a multilevel human cadaveric cervical model. Neurosurgical Focus. 2004;17:22-29.

9. Steinmetz MP, Benzel EC, Apfelbaum RI. Subsidence and dynamic cervical spine stabilization. Spine surgery: Techniques, complication avoidance, and management, $2^{\text {nd }}$ Ed. Churchill Livingstone Publishing, Philadelphia, PA. Chapter 113: 1477-1485.

10. Wolff's Law. Encyclopedia Britannica. 2008. Encyclopedia Britannica Online. Accessed 10 Apr. 2008. http://www.britannica.com/EBchecked/topic/481191/Wolffs-law.

11. Panjabi MM, Krag M, Summers D, et al. Biomechanical time tolerance of fresh cadaveric spine specimens. J Orthop Res. 1985;3:292-300.

12. Reinsel T, Krag M. Cervical spine biomechanics. Surgery of the cervical spine. 1994. Saunders College Publishing, Philadelphia, PA. Chapter 3:75-94.

13. Pseudarthrosis. Encyclopedia Britannica. 2008. Encyclopedia Britannica Online. Accessed 10 Apr. 2008. http://www.britannica.com/EBchecked/topic/481191/pseudarthrosis. 
14. Stulik J, Pitzen TR, Steudel WI, et al. Fusion and failure following anterior cervical plating with dynamic or rigid plates: 6-months results of a multi-centric, prospective, randomized, controlled study. Eur Spine J. 2007 Oct;16(10):1689-1694.

15. Apjlbaum RI, Daily AT, Barbra J. Clinical experience with a new load sharing dynamic anterior cervical plate. Spine Universe. Epub 20 Feb. 2007. Accessed 14 Mar. 2008. http://www.spineuniverse.com/displayarticle.php/article425.html.

16. Leon SP, DiAngelo DJ, Foley KT, et al. Spinal implant attributes: Dynamic spine fixation. Spine surgery: Techniques, complication avoidance, and management, $2^{\text {nd }}$ Ed. Churchill Livingstone Publishing, Philadelphia, PA. Chapter 108: 1430-1433. 
APPENDICES 
Appendix A:

Normalized Flexibility 

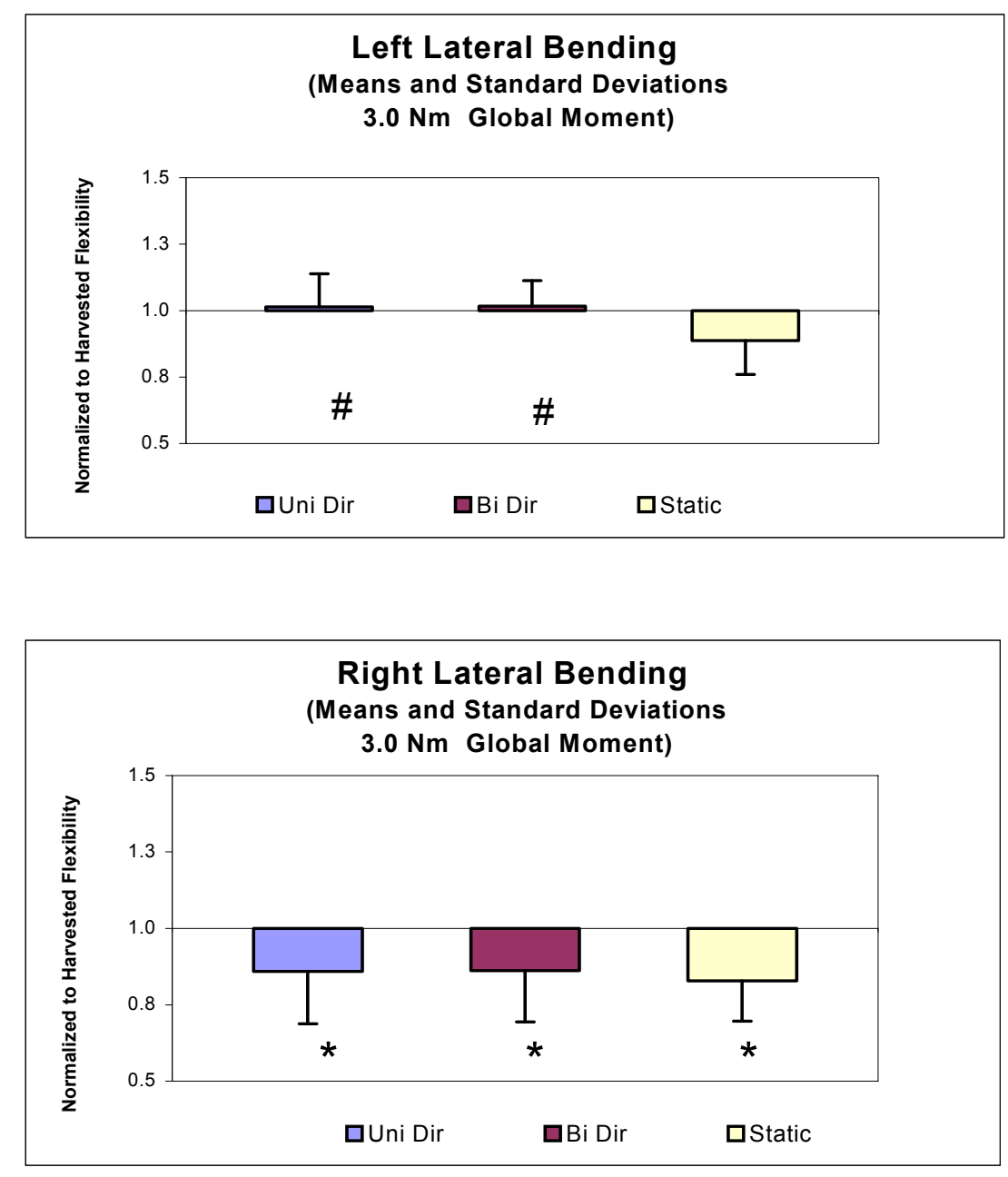

Figure A.1 Normalized Flexibility for Left and Right Lateral Bending. (* Signifies significant difference from the harvested condition. \# Signifies significant difference from the static condition. $\Psi$ Signifies significant difference between the dynamic conditions.) 

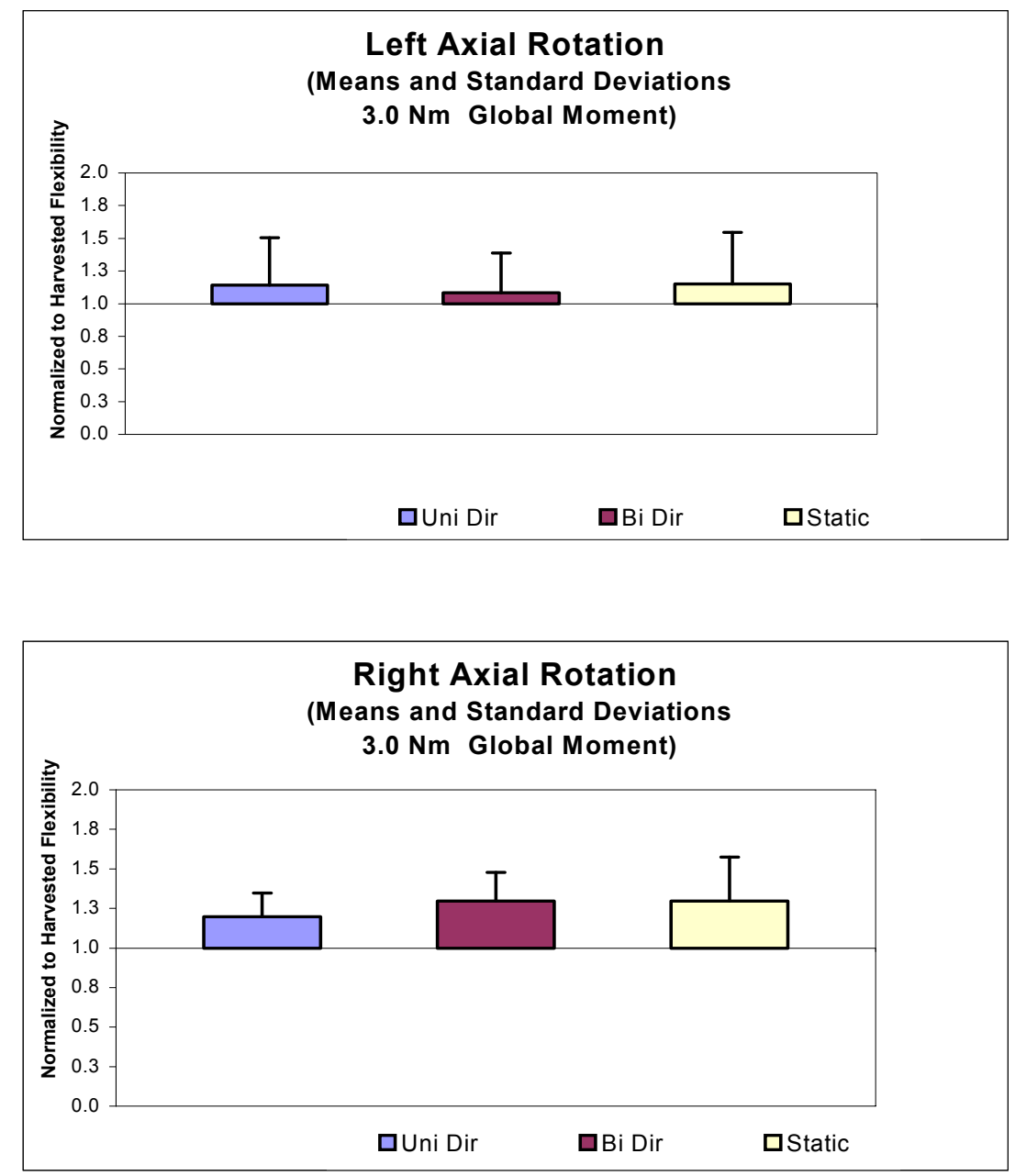

Figure A.2 Normalized Flexibility for Left and Right Axial Rotation. (* Signifies significant difference from the harvested condition. \# Signifies significant difference from the static condition. $\Psi$ Signifies significant difference between the dynamic conditions.) 
Appendix B:

Normalized Motion 

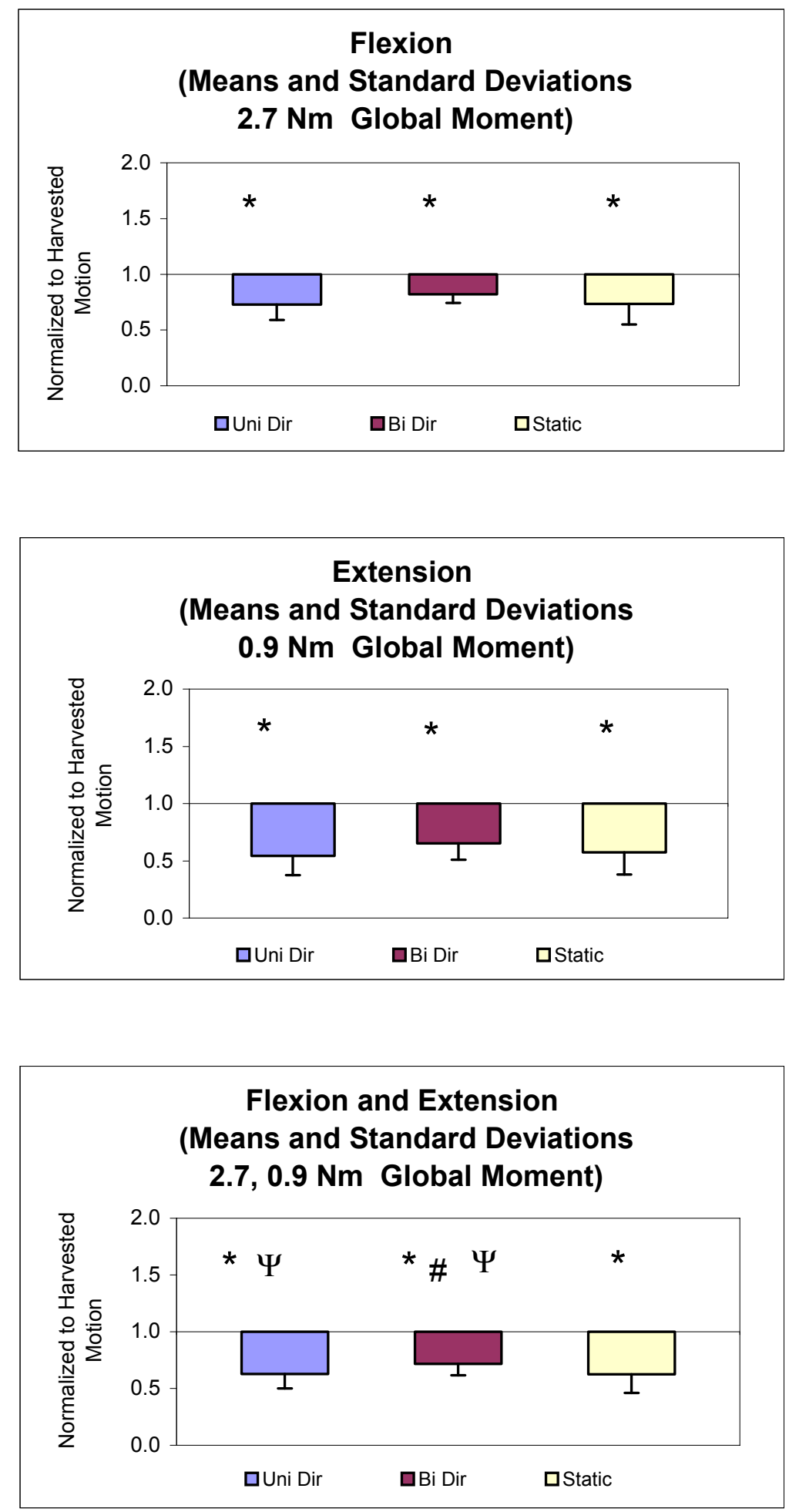

Figure B.1 Normalized Motion for Flexion and Extension. (* Signifies significant difference from the harvested condition. \# Signifies significant difference from the static condition. $\Psi$ Signifies significant difference between the dynamic conditions.) 

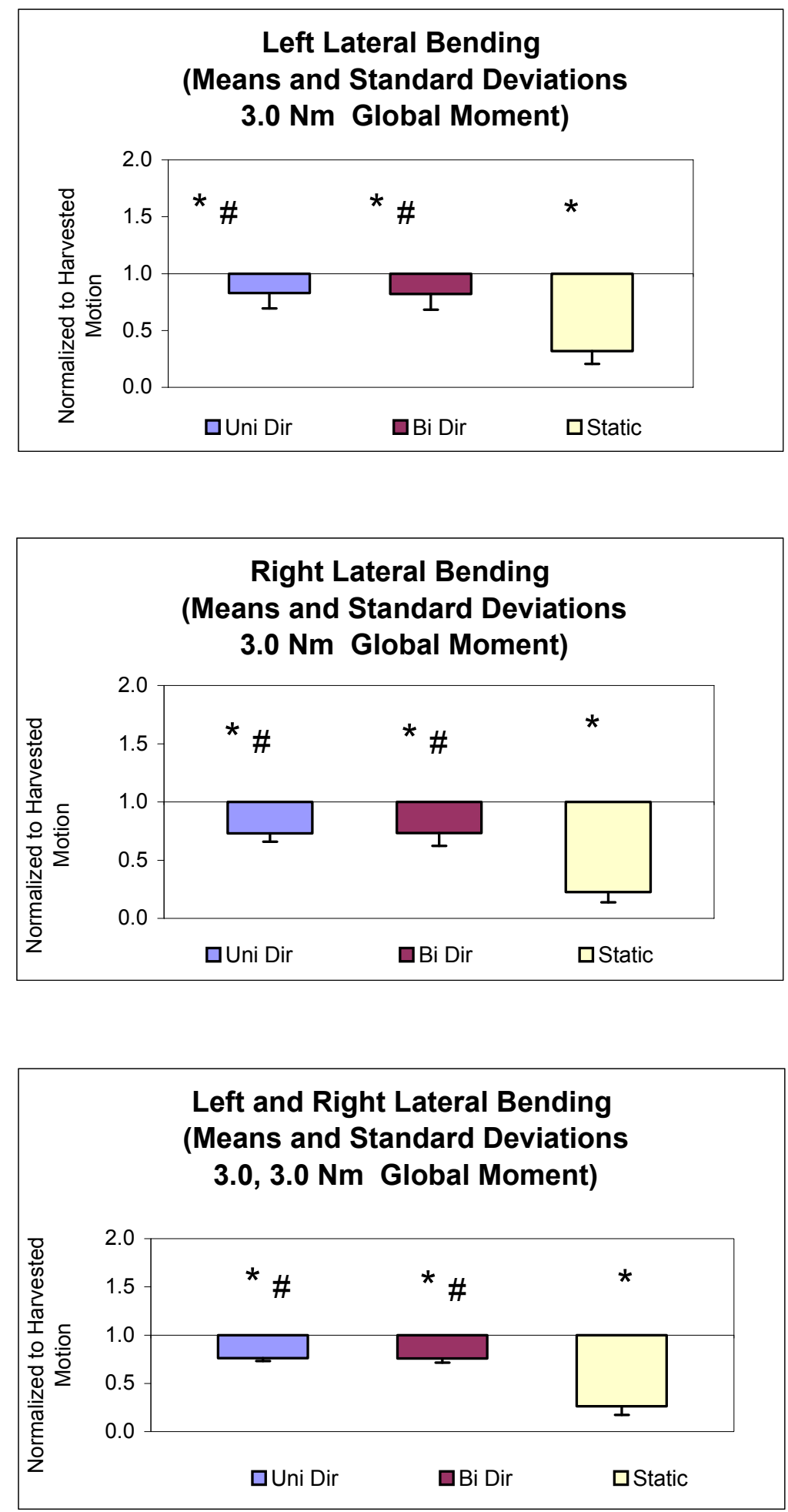

Figure B.2 Normalized Motion for Left and Right Lateral Bending. (* Signifies significant difference from the harvested condition. \# Signifies significant difference from the static condition. $\Psi$ Signifies significant difference between the dynamic conditions.) 

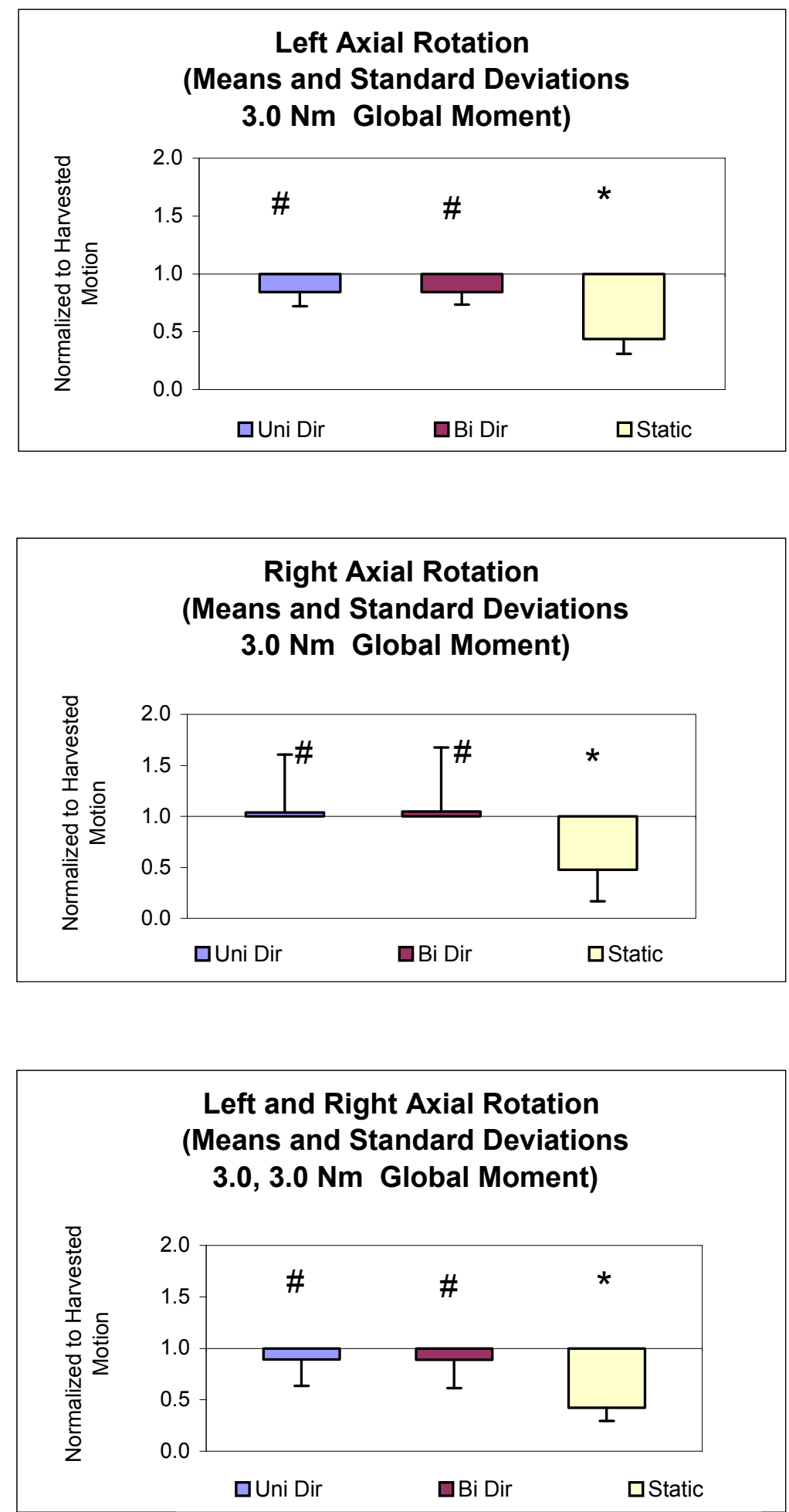

Figure B.3 Normalized Motion for Left and Right Axial Rotation. (* Signifies significant difference from the harvested condition. \# Signifies significant difference from the static condition. $\Psi$ Signifies significant difference between the dynamic conditions.) 
Appendix C:

Motion Segment Unit Percent Contribution 


\section{Flexion}
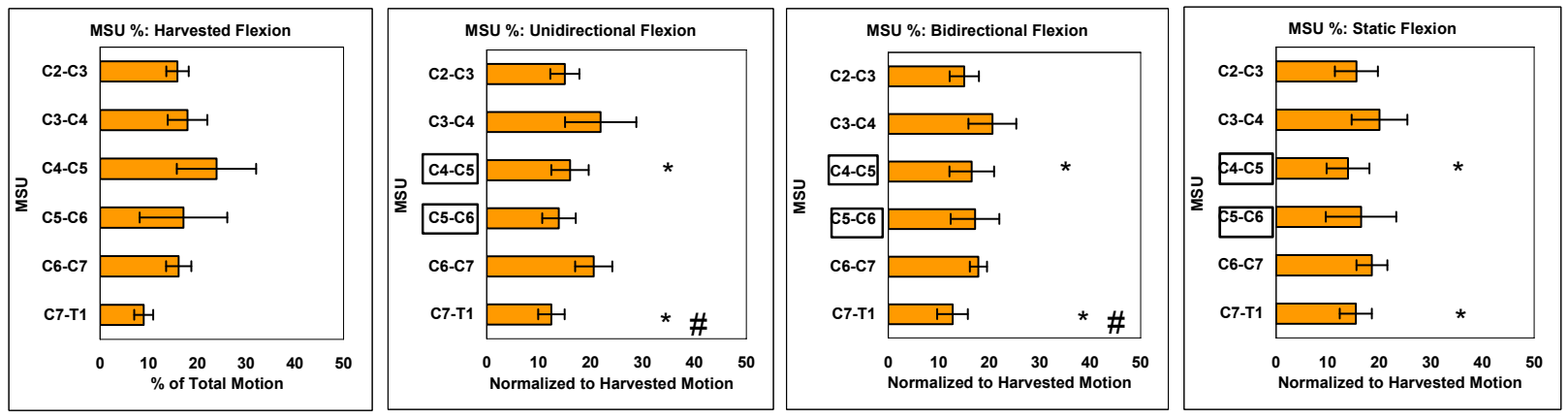

\section{Extension}
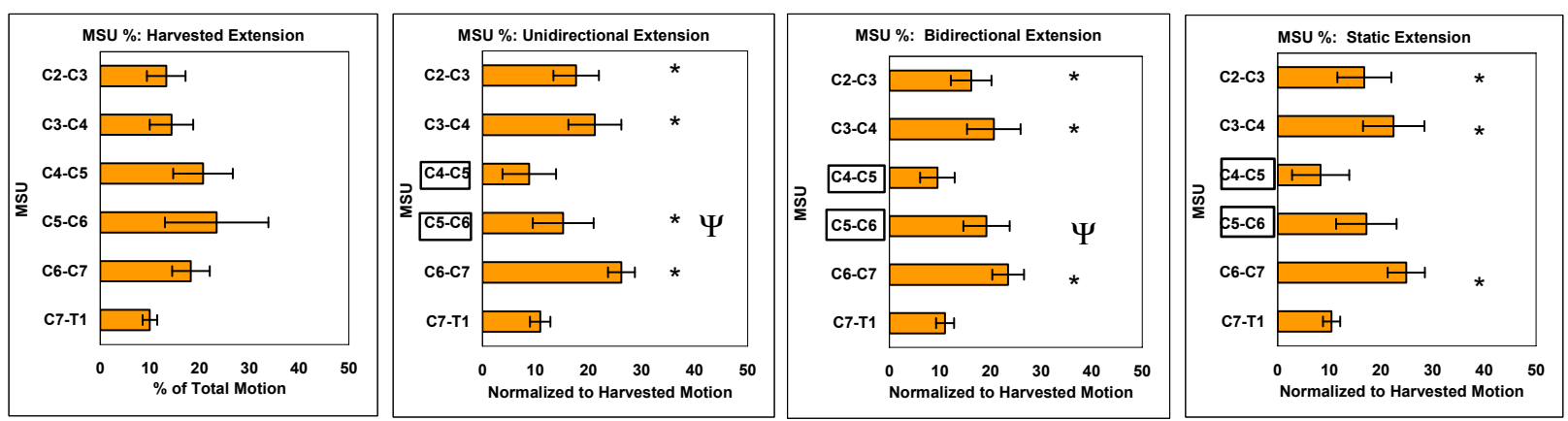

\section{Flexion and Extension}
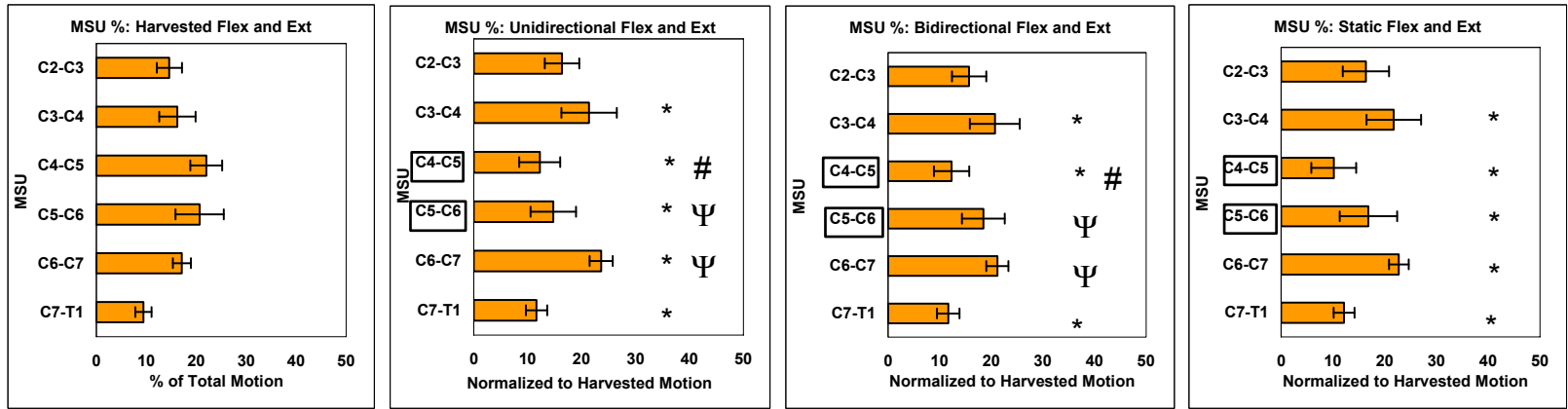

Figure C.1 Motion Segment Unit Percent Contribution for Flexion and Extension.

( Signifies significant difference from the harvested condition. \# Signifies significant difference from the static condition. $\Psi$ Signifies significant difference between the dynamic conditions.) 


\section{Left Lateral Bending}
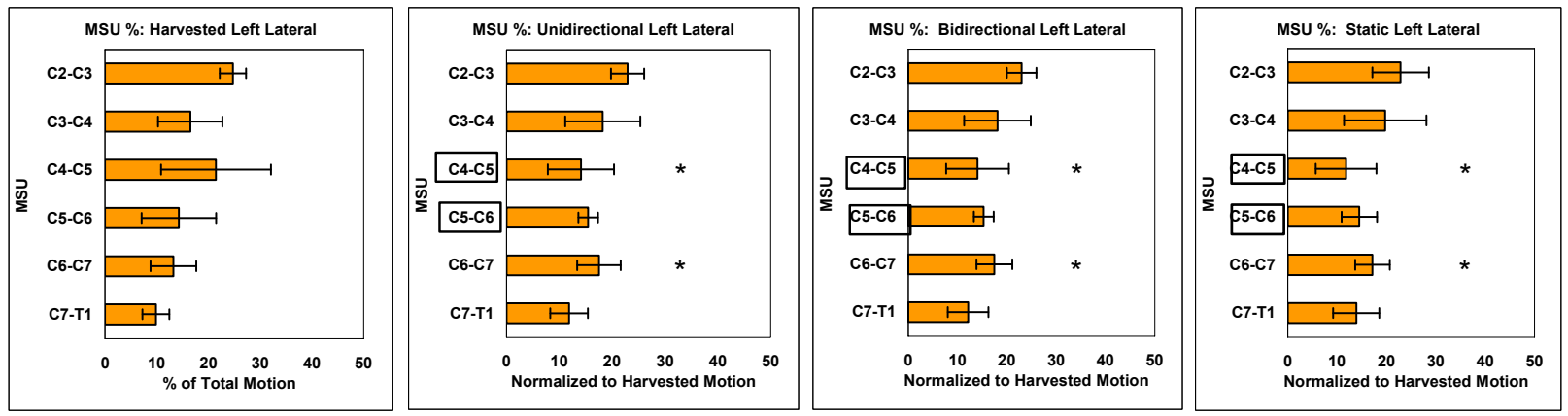

Right Lateral Bending
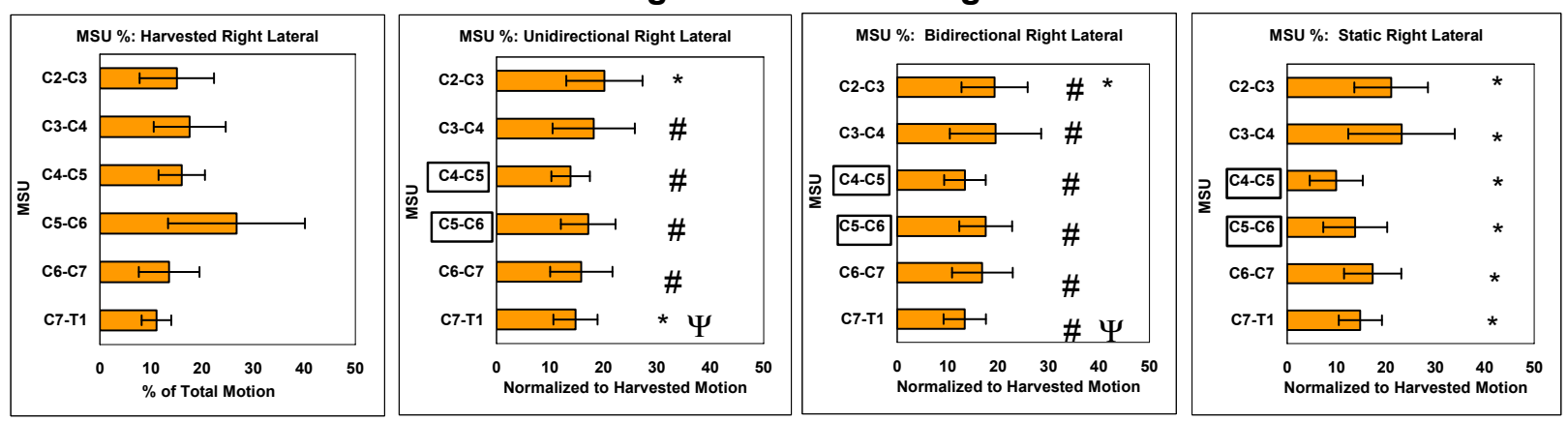

\section{Left and Right Lateral Bending}
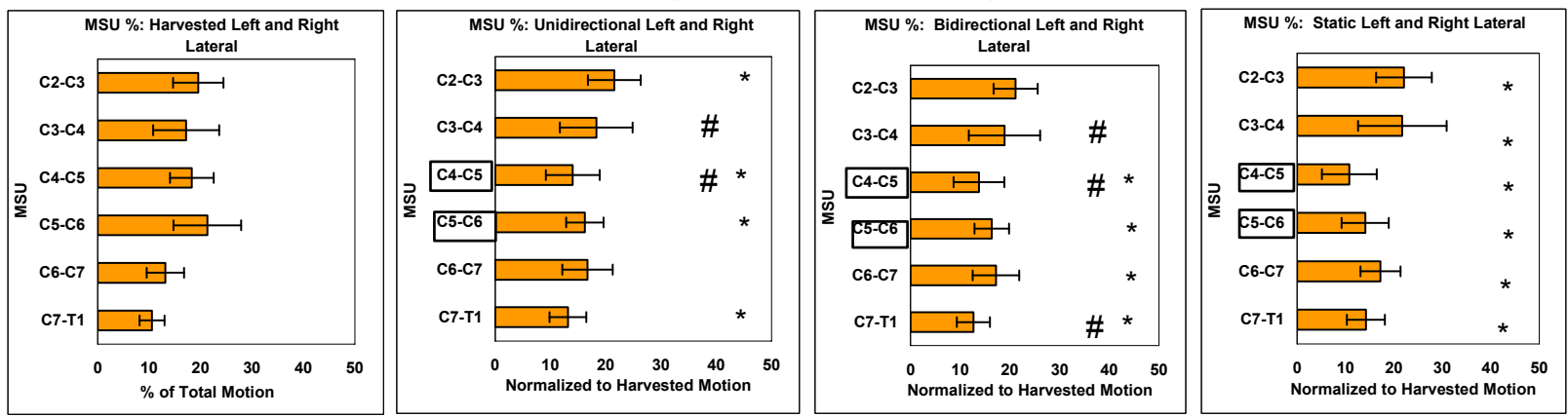

Figure C.2 Motion Segment Unit Percent Contribution for Left and Right Lateral Bending. (* Signifies significant difference from the harvested condition. \# Signifies significant difference from the static condition. $\Psi$ Signifies significant difference between the dynamic conditions.) 


\section{Left Axial Rotation}
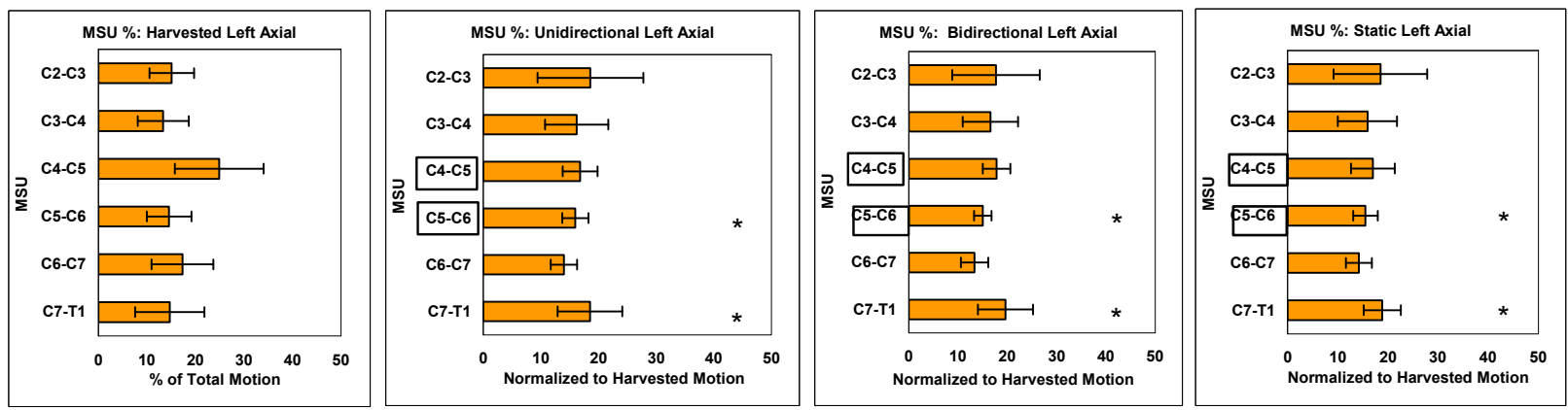

\section{Right Axial Rotation}
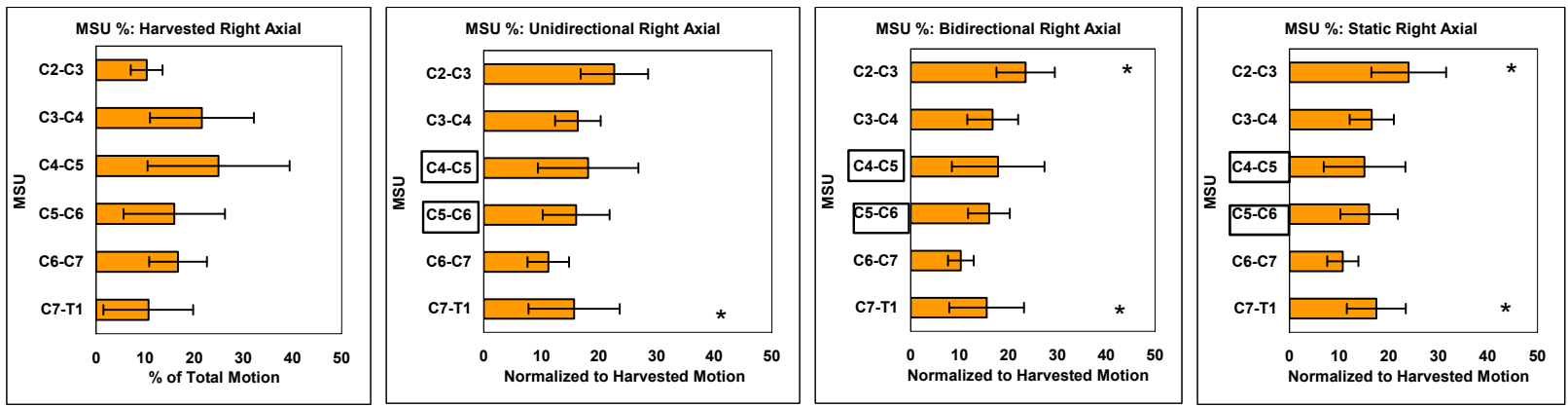

\section{Left and Right Axial Rotation}
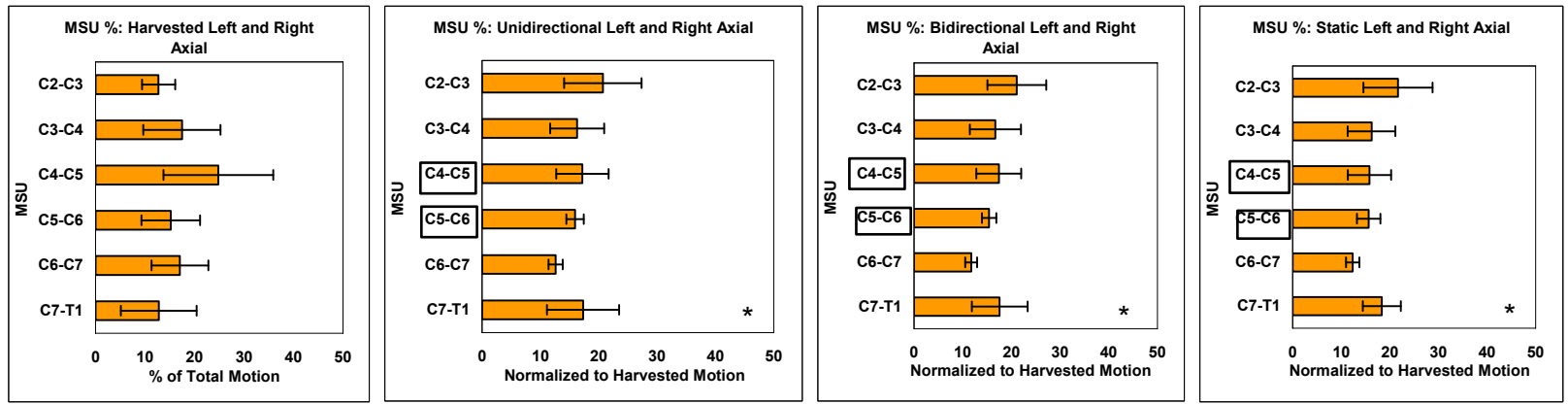

Figure C.3 Motion Segment Unit Percent Contribution for Left and Right Axial Rotation. (* Signifies significant difference from the harvested condition. \# Signifies significant difference from the static condition. $\Psi$ Signifies significant difference between the dynamic conditions.) 

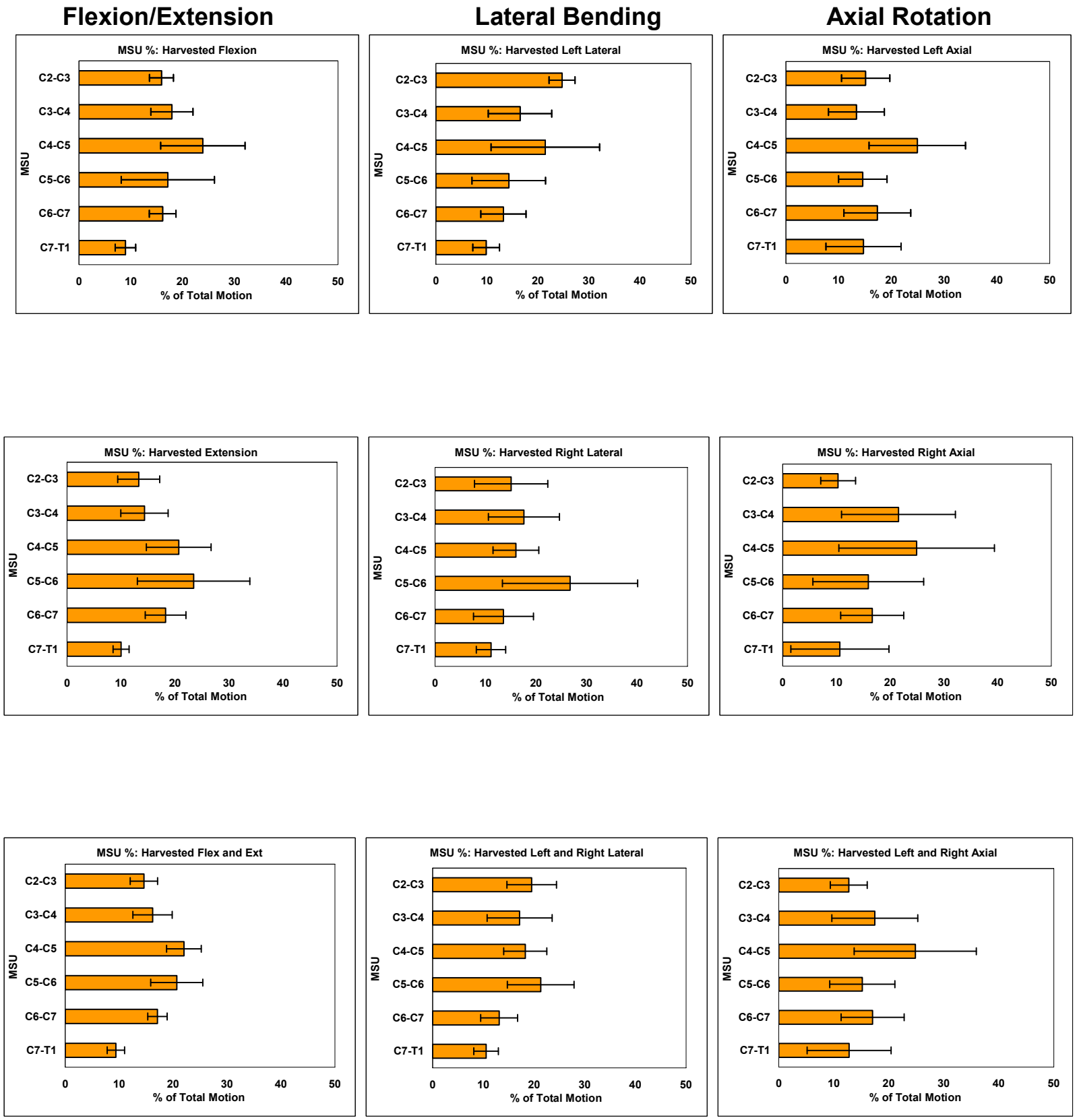

Figure C.4 Motion Segment Unit Percent Contribution for Harvested Condition Only. 
Appendix D:

Peak Graft Loads 

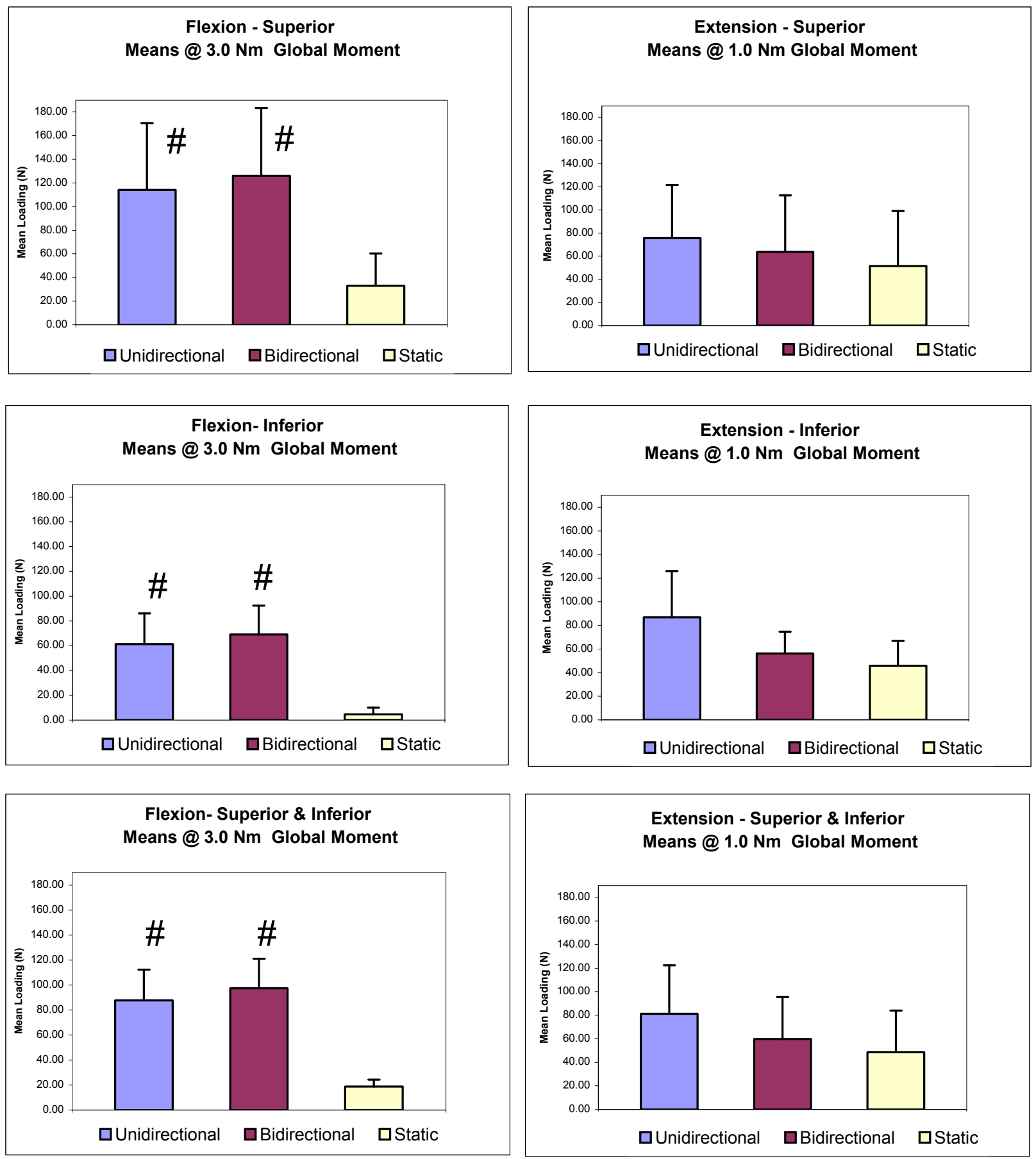

Figure D.1 Peak Graft Loads for Flexion and Extension. (\# Signifies significant difference from the static condition. $\Psi$ Signifies significant difference between the dynamic conditions.) 

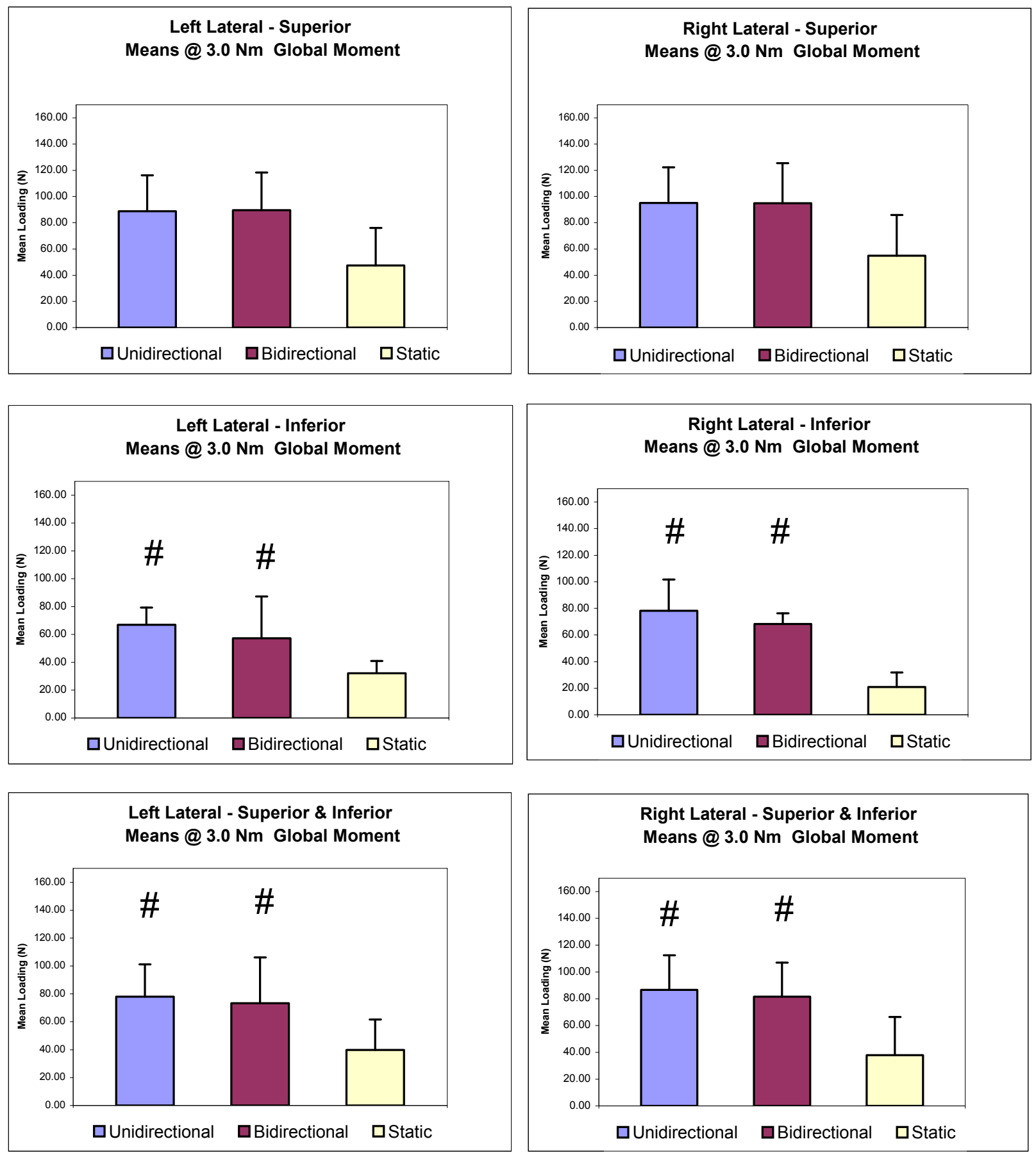

Figure D.2 Peak Graft Loads for Left and Right Lateral Bending. (\# Signifies significant difference from the static condition. $\Psi$ Signifies significant difference between the dynamic conditions.) 

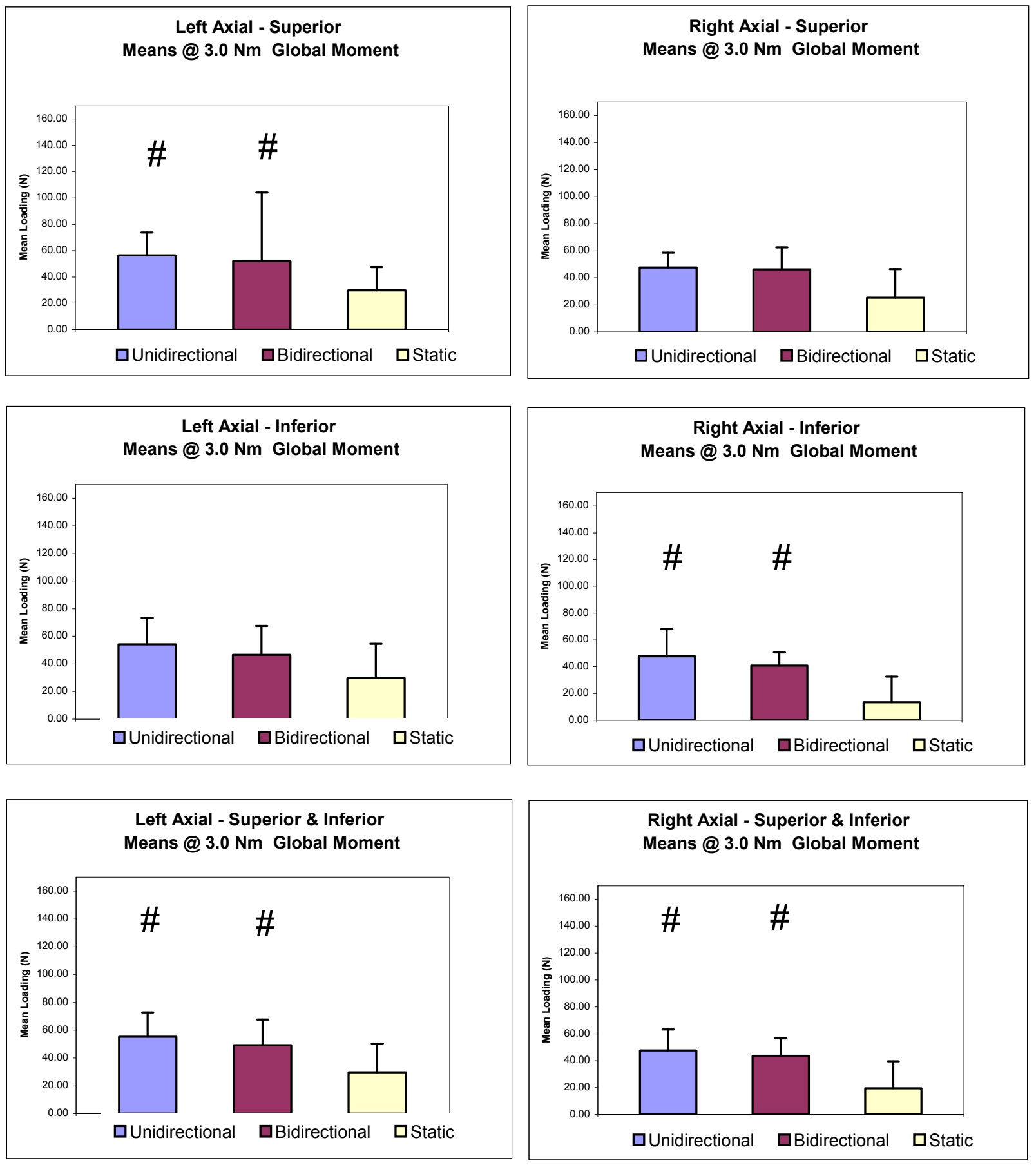

Figure D.3 Peak Graft Loads for Left and Right Axial Rotation. (\# Signifies significant difference from the static condition. $\Psi$ Signifies significant difference between the dynamic conditions.) 
Appendix E:

Change in Graft Loads 

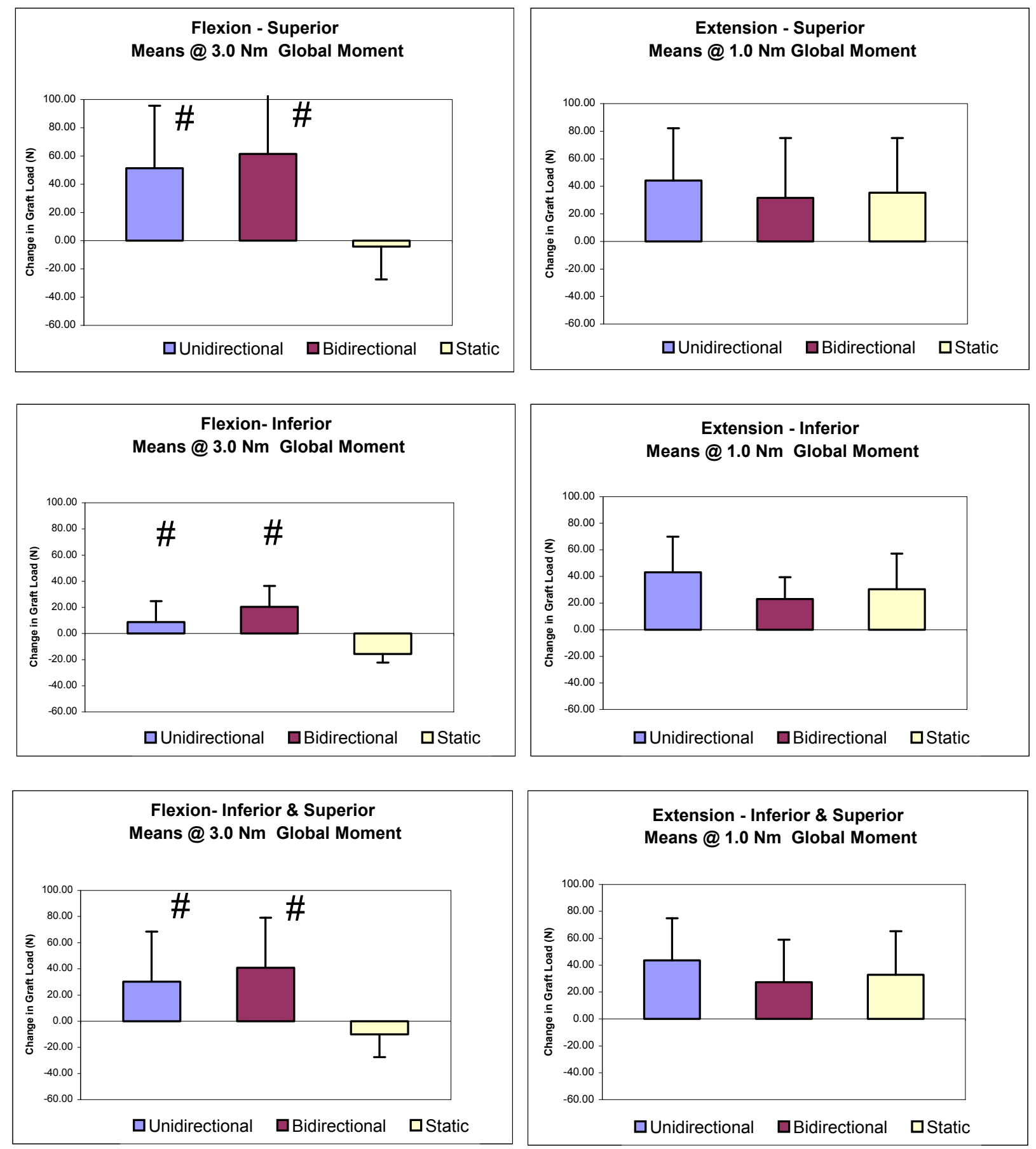

Figure E.1 Change in Graft Loads for Flexion and Extension. (\# Signifies significant difference from the static condition. $\Psi$ Signifies significant difference between the dynamic conditions.) 

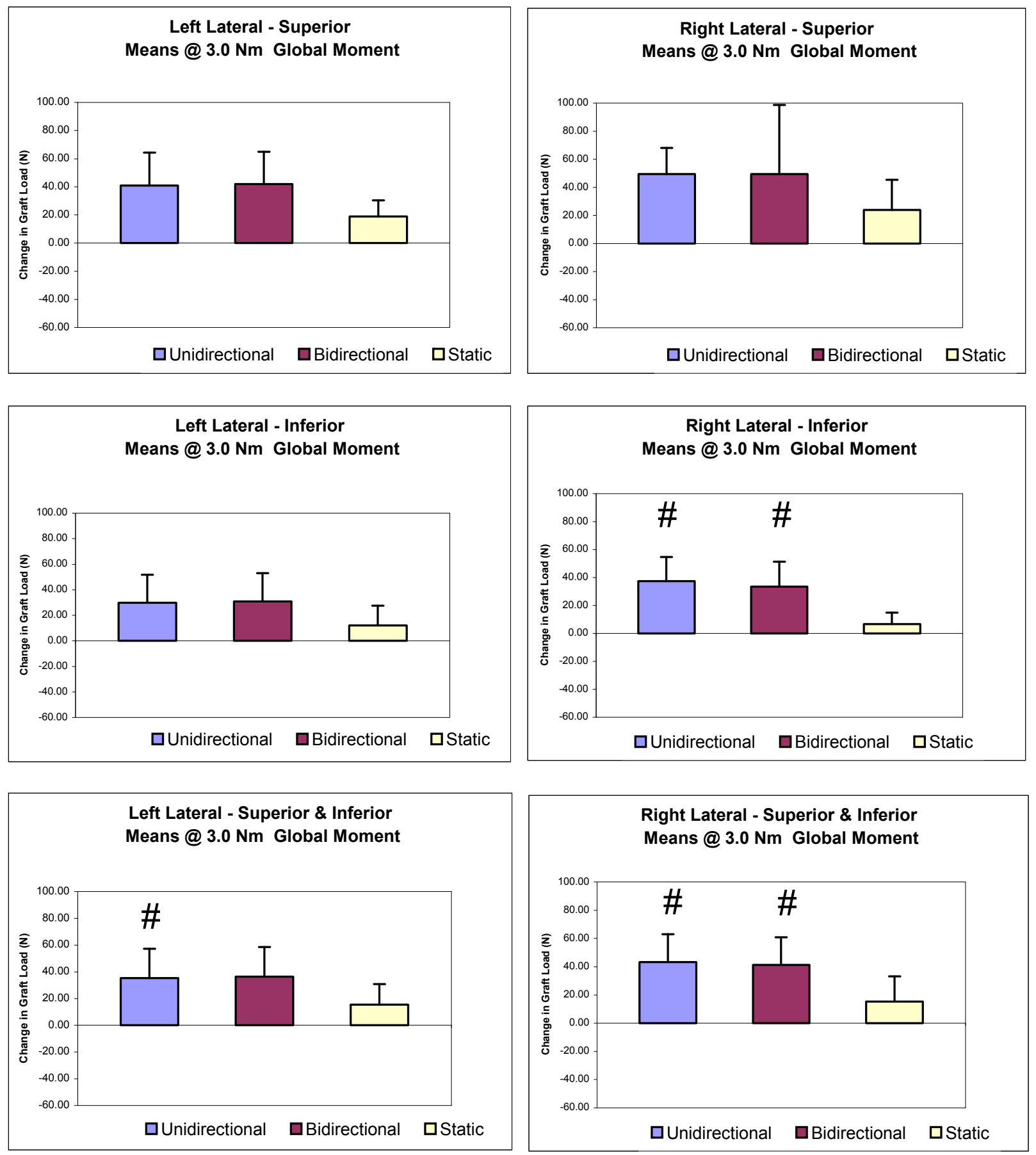

Figure E.2 Change in Graft Loads for Left and Right Lateral Bending. (\# Signifies significant difference from the static condition. $\Psi$ Signifies significant difference between the dynamic conditions.) 

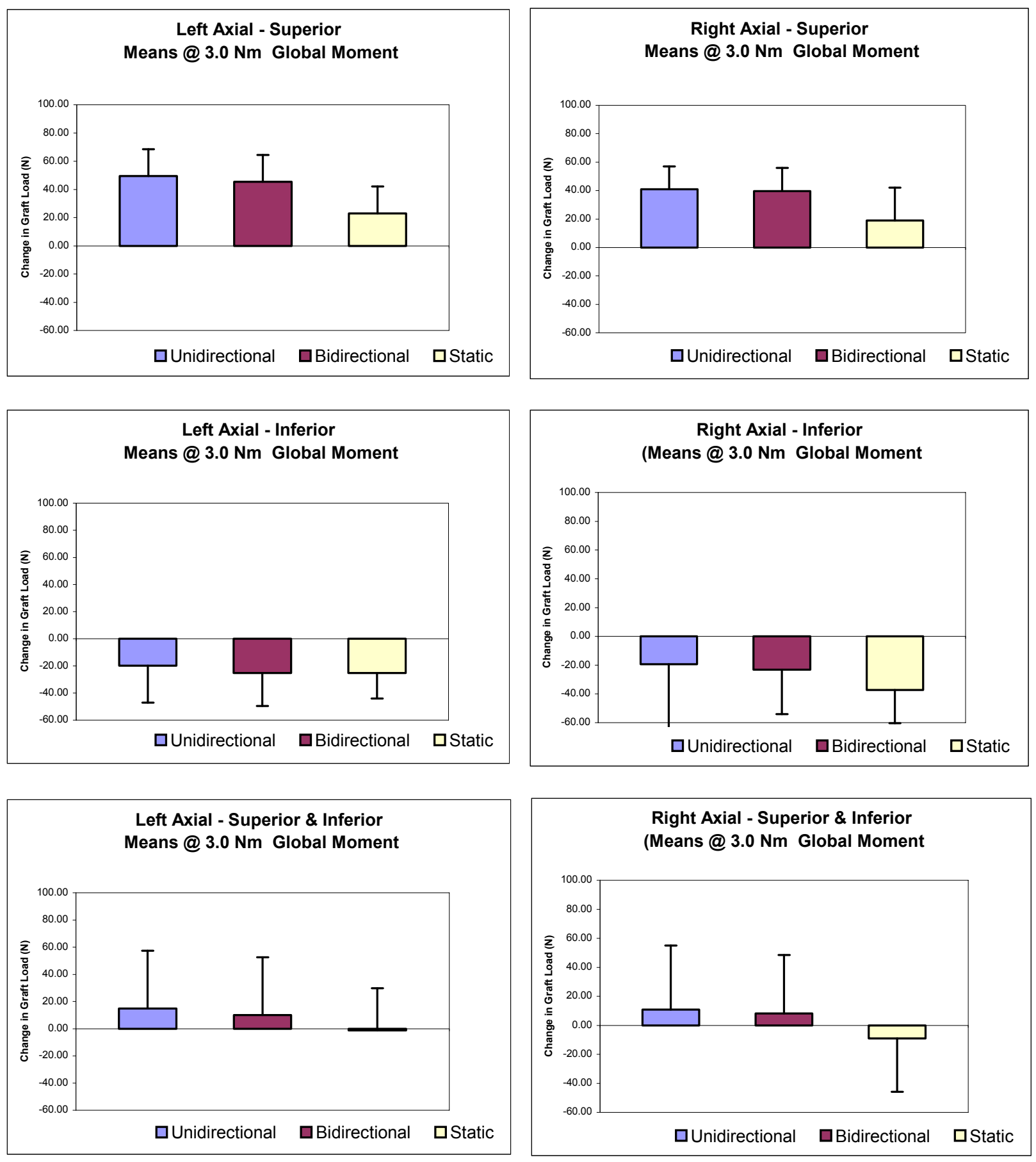

Figure E.3 Change in Graft Loads for Left and Right Axial Rotation. (\# Signifies significant difference from the static condition. $\Psi$ Signifies significant difference between the dynamic conditions.) 
Appendix F:

Graft Preloads 

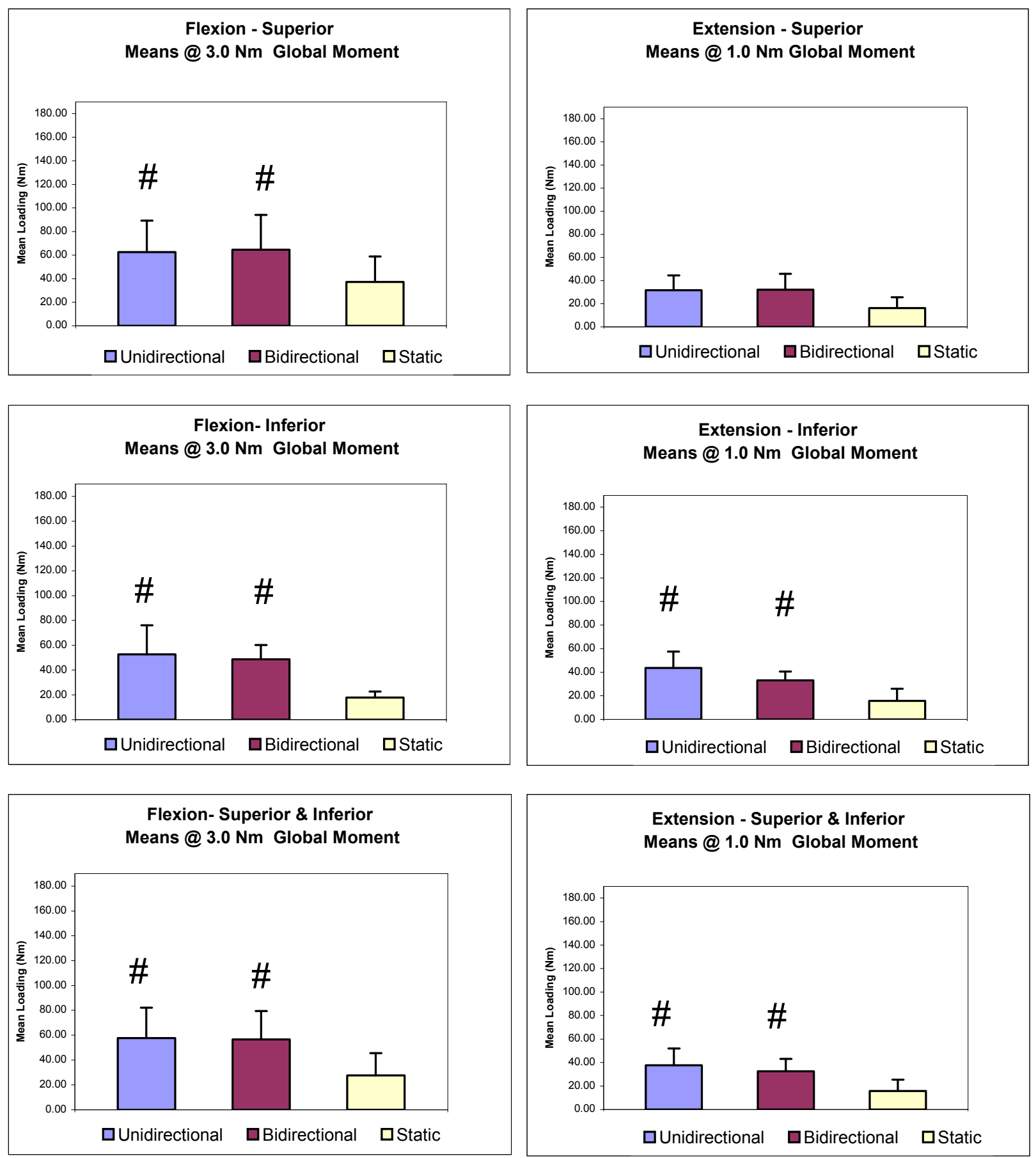

Figure F.1 Graft Preloads for Flexion and Extension. (\# Signifies significant difference from the static condition. $\Psi$ Signifies significant difference between the dynamic conditions.) 

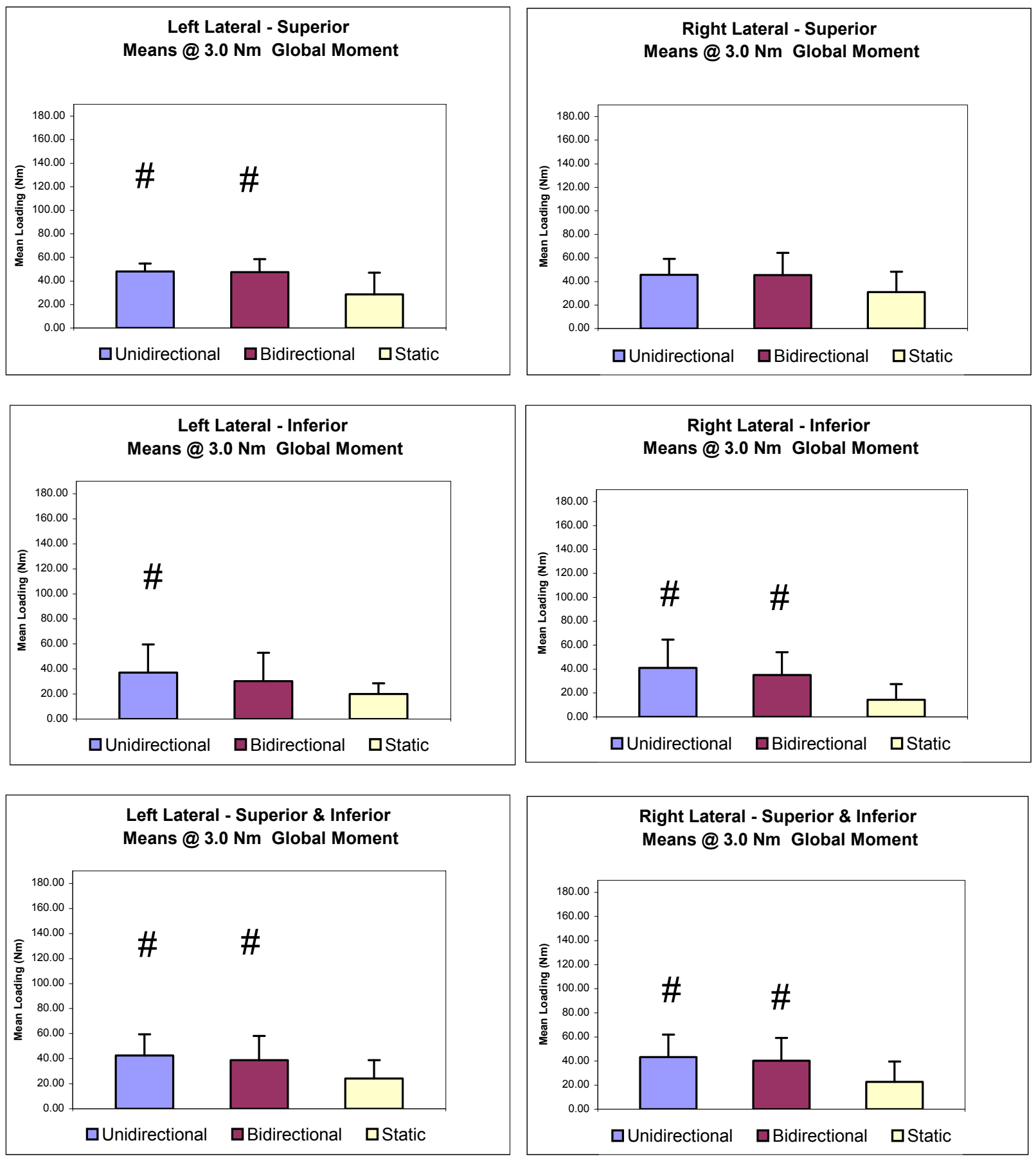

Figure F.2 Graft Preloads for Left and Right Lateral Bending. (\# Signifies significant difference from the static condition. $\Psi$ Signifies significant difference between the dynamic conditions.) 

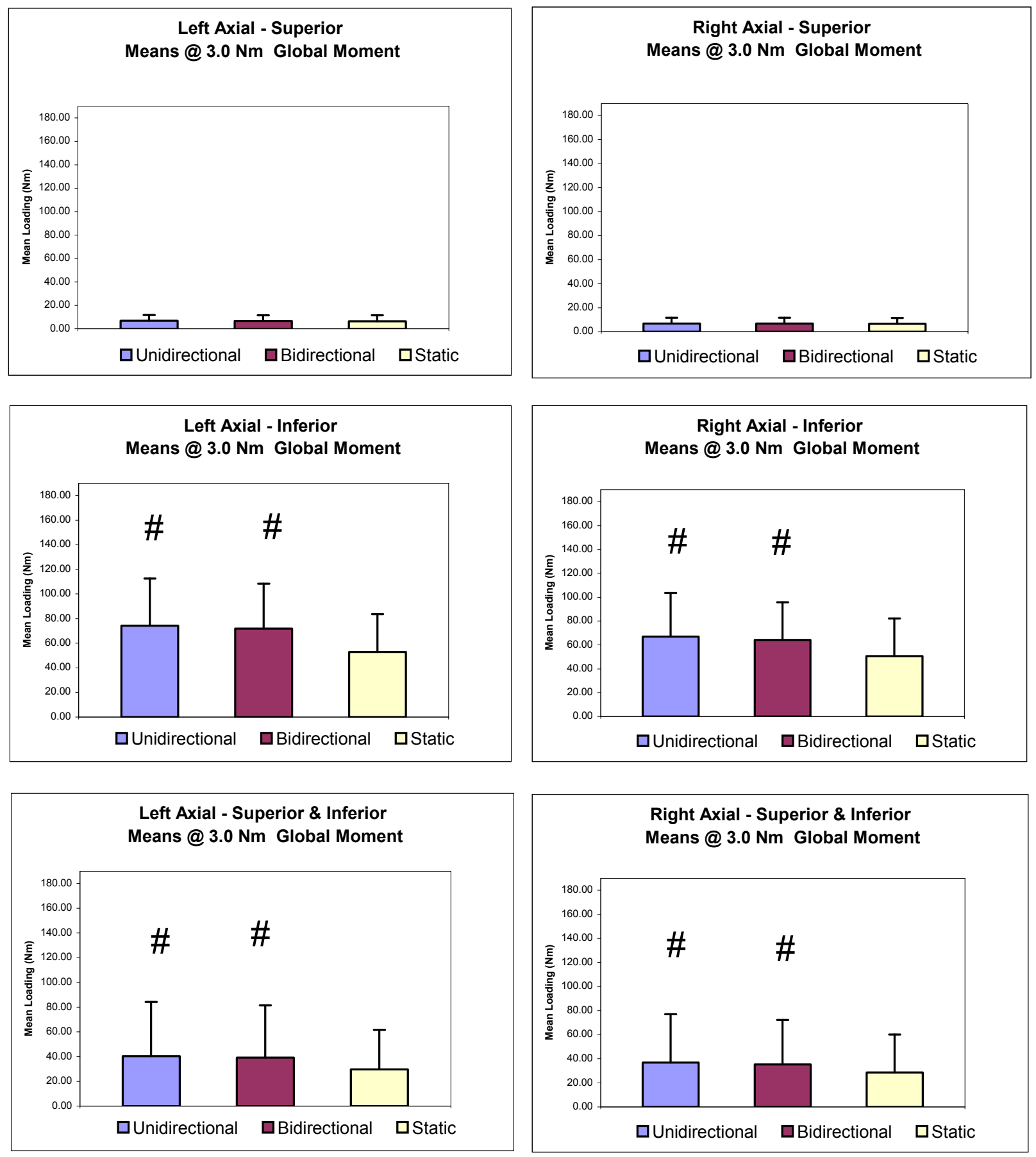

Figure F.3 Graft Preloads for Left and Right Axial Rotation. (\# Signifies significant difference from the static condition. $\Psi$ Signifies significant difference between the dynamic conditions.) 


\section{VITA}

Jason Roberson was born in Orlando, Florida on July 5, 1977 and grew up in nearby Kissimmee, Florida. He graduated with honors from Osceola High School, class of 1995. He then spent a six year enlistment in the United States Navy, where he served as a Nuclear reactor plant operator onboard the USS Nimitz (CVN-68), where he circled the globe, assisted with a nuclear refueling and overhaul, and had some of the best experiences of his professional career. In 2002, he finished his undergraduate degree in Mechanical Engineering Technology at Old Dominion University, Norfolk, Virginia, where he met his future wife. He spent several years in industry as a process and manufacturing engineer, before he continued his education at the University of Tennessee Graduate Health Science Center. He currently lives in Orlando, Florida with his wife Jessica, and his son Jacob. 